\title{
Electronic Structure and Absorption Spectra of Biferrocenyl and Bisfulvalenide Diiron Radical Cations: Detection and Assignment of New Low-Energy Transitions
}

\author{
Ralf Warratz, ${ }^{[a]}$ Hanane Aboulfadl, ${ }^{[b]}$ Thomas Bally, ${ }^{* b]}$ and Felix Tuczek*[a]
}

\begin{abstract}
UV-visible/near-IR (NIR)/ mid-IR (MIR) solution, solid-state, and matrix-isolation electronic absorption spectra of the $\mathrm{Fe}^{\mathrm{II}}-\mathrm{Fe}^{\mathrm{III}}$ mixed-valent homobimetallic compounds biferrocenyl triiodide (1) and 1', $1^{\prime \prime \prime}$-diethylbiferrocenyltriiodide (2) reveal the presence of a low-energy transition in the MIR region that has not been reported before. The new absorption feature and the known NIR band are both assigned to intervalence charge-transfer (IVCT) transitions. To obtain insight
\end{abstract}

into the electronic structures of $\mathbf{1}$ and 2, DFT calculations with the BP86 functionals and different basis sets have been performed. Based on the molecular orbital scheme of cation $\mathbf{1}$, one band corresponds to the transition between the highest occupied $\mathrm{d}_{x^{2}-y^{2}}$ or-

Keywords: electronic structure iron • mixed-valent compounds • optical absorption spectroscopy • radical ions

\section{Introduction}

Mixed-valent transition-metal dimers have been and continue to be of interest for the investigation of inner-sphere electron transfer. On the basis of their tremendous impact on this research area, $\mathrm{Fe}^{\mathrm{II}}-\mathrm{Fe}^{\mathrm{III}}$ bimetallocenes ${ }^{[1-8]}$ can in fact be considered as the organometallic counterparts of the Creutz-Taube ion. A phenomenon that is exclusively associated with this class of compounds and that has aroused considerable interest over the years is the transition from a charge-localized state at low temperatures to a delocalized

[a] Dr. R. Warratz, Prof. Dr. F. Tuczek

Institut für Anorganische Chemie

Christian Albrechts Universität Kiel

Otto Hahn Platz 6/7, 24098 Kiel (Germany)

Fax: (+49) 431-880-1520

E-mail: ftuczek@ac.uni-kiel.de

[b] H. Aboulfadl, Prof. T. Bally

Département de Chimie

Université de Fribourg

Chemin du Musée 9, 1700 Fribourg (Switzerland)

Fax: (+41)26-300-97-37

E-mail: Thomas.Bally@unifr.ch bitals on the two iron centers, whereas the other one is assigned to a transition from a lower-lying $\mathrm{d}_{z^{2}}$ orbital to the $\mathrm{d}_{x^{2}-y^{2}}$ orbital. For comparison, the doubly bridged bisfulvalenide diiron cation (3) has been investigated by optical absorption spectroscopy and DFT calculations. The experimental and theoretical results are discussed with respect to the degree of electron localization/delocalization in these systems. state at higher temperatures. This effect, which has been discovered and primarily investigated by means of Mössbauer spectroscopy, ${ }^{[2]}$ renders these systems particularly well suited to study the dependence of electron-transfer rates on small geometric changes, a subject that is of general interest to all areas of chemistry, biology, and physics.

The charge-localization-delocalization transition may be exemplified on ferrocenyl-ferrocenium (biferrocenyl) triiodide, $\mathrm{Fc}_{2} \mathrm{I}_{3}$ (actually $\mathrm{Fc}_{2}{ }^{-+} \mathrm{I}_{3}{ }^{-} ; \mathbf{1}$ ) and $1^{\prime}, 1^{\prime \prime \prime}$-diethylbiferrocenyl triiodide, $(\mathrm{FcEt})_{2} \mathrm{I}_{3}(\mathbf{2})$, which have been studied in detail by Mössbauer spectroscopy and other techniques. ${ }^{[1-8]}$

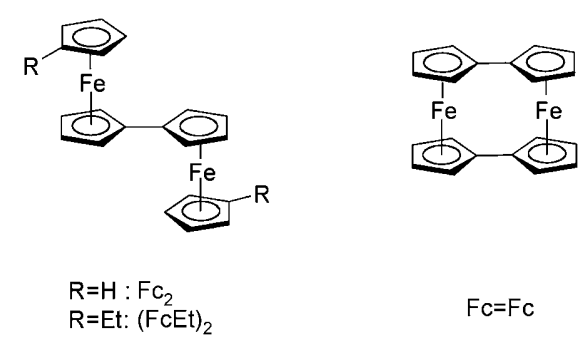

In the solid state, $\mathrm{Fc}_{2} \mathrm{I}_{3}$ is charge localized between 4.2 and $300 \mathrm{~K}$, and exhibits two discrete quadrupole doublets 
for $\mathrm{Fe}^{\mathrm{II}}$ and $\mathrm{Fe}^{\mathrm{III}}$. At a critical temperature $\left(T_{\mathrm{c}}\right)$ of $360 \mathrm{~K}$, a transition to a charge-delocalized state occurs, which is evident from the appearance of a single quadrupole doublet. ${ }^{[9,10]}$ In contrast, for $(\mathrm{FcEt})_{2} \mathrm{I}_{3}$ the charge-localizationdelocalization transition occurs at $T_{\mathrm{c}}=275 \mathrm{~K}$. At lower temperature the system is localized (two quadrupole doublets) and at higher temperature delocalized (one quadrupole doublet). Importantly, the Mössbauer spectra do not exhibit line broadening at any temperature, in particular also not in the vicinity of $T_{\mathrm{c}}$. This indicates that electronic relaxation is always rapid on the Mössbauer time scale, a result that has been difficult to understand for a long time. ${ }^{[11]}$

A variety of theoretical approaches have been developed to interpret these phenomena. ${ }^{[12,13,14]}$ In the vibronic model of Varret and co-workers, which has been further elaborated by Klokishner et al. ${ }^{[15]}$ the charge-localization-delocalization transitions are attributed to cooperative interactions between the bimetallocene molecules in the crystalline solid, which modulate the energy difference $\left(W_{\mathrm{d}}\right)$ between the local minima of the double-well potentials of the individual dimers. At or above $T_{\mathrm{c}}$ the energy difference $W_{\mathrm{d}}$ becomes zero. The $\mathrm{Fe}^{\mathrm{II}}-\mathrm{Fe}^{\mathrm{III}}$ units thus can be described as symmetrical class II-III mixed-valent dimers (according to the Robin and Day classification scheme) ${ }^{[16,17]}$ and in the presence of fast relaxation a charge-delocalized state (exhibiting, for example, one Mössbauer doublet) is observed. Below $T_{\mathrm{c}}$ the potential becomes asymmetric and $W_{\mathrm{d}}$ may become large compared to $k T$, leading to a charge-ordered state. In this case two distinct Mössbauer doublets can be observed, one mostly deriving from $\mathrm{Fe}^{\mathrm{III}}$ and the other one mostly from $\mathrm{Fe}^{\mathrm{II}}$. The cooperative interactions that trigger the transition from the charge-delocalized to the charge-ordered state sensitively depend on the substituents ${ }^{[9,12]}$ and the counterions ${ }^{[18,19]}$ of the biferrocenyl radical cations, thus explaining the observation of different $T_{\mathrm{c}}$ values for different systems.

Besides Mössbauer spectroscopy, a range of physical and spectroscopic methods have been employed to study the electronic and geometric structure as well as structural features of these intriguing systems. ${ }^{[1]}$ In particular, infrared and EPR spectroscopies were applied to detect temperature-dependent phenomena. Thereby it was found that the transition between static and fluxional behavior could occur at different temperatures, depending on the characteristic time scale of the method. Moreover, heat capacity measurements were conducted to monitor the enthalpic changes associated with the charge-localization-delocalization transition. ${ }^{[20]}$ Important information on the structural consequences of this transition was also obtained by X-ray crystallography. This technique conclusively demonstrated the presence of an asymmetric distortion of the individual dimers below $T_{\mathrm{c}}$ that disappears at $T_{\mathrm{c}}$ and higher temperatures. ${ }^{[21]}$

In spite of the wealth of structural, physical, and spectroscopic data being available for $\mathrm{Fe}^{\mathrm{II}}-\mathrm{Fe}^{\mathrm{III}}$ bimetallocenes, relatively little information exists with respect to their optical absorption properties. This is surprising because for other mixed-valence systems the detection and detailed analysis of the intervalence charge transfer (IVCT) transition has played a decisive role in their physico-chemical characterization. ${ }^{[22,23]}$ An IVCT transition has been detected for biferrocenyl cations and has been interpreted on the basis of the Piepho-Krausz-Schatz (PKS) model. ${ }^{[2]}$ However, no temperature dependence was recorded and no good fit of the room-temperature data was obtained. ${ }^{[25]}$ We therefore decided to reinvestigate the radical cations of $\mathrm{Fc}_{2}$ and $(\mathrm{FcEt})_{2}$ by means of optical absorption spectroscopy, both in the solidstate and in solution. Here the results of this study are presented. To obtain the full spectroscopic information, the wavelength range is extended to the mid-IR (MIR) range where, in addition to the known near-IR (NIR) transition, a new absorption feature is detected.

To interpret the optical spectra, in particular the unexpected presence of two absorption features in the lowenergy range, electronic structure calculations were performed. These calculations allowed us to assign these features to two spectrally overlapping transitions. For comparison with the singly bridged biferrocenyl cations $\mathrm{Fc}_{2}{ }^{+}$and $(\mathrm{FcEt})_{2}{ }^{+}$the corresponding doubly bridged bisfulvalenide diiron cation $\mathrm{Fc}=\mathrm{Fc}^{*+}$ was investigated by the same methods. Importantly, this dimer also exhibits a two-peak MIR transition, a phenomenon that has been known and interpreted before. ${ }^{[6,25,26,27]}$ Moreover, Mössbauer spectroscopy results showed that the bisfulvalenide cation is always delocalized and no localization-delocalization transitions exist in this type of molecule. Herein, the electronic structure of this dimer is also evaluated by means of DFT calculations and compared with that of the singly bridged counterparts. A forthcoming study will be devoted to a detailed analysis of the band shapes of the observed low-energy absorption bands, and in particular, their temperature dependence. ${ }^{[28]}$

\section{Experimental Section}

Materials: Biferrocene $\left(\mathrm{Fc}_{2}\right),{ }^{[12,29]} 1^{\prime}, 1^{\prime \prime \prime}$-diethylferrocenyl biferrocene $\left((\mathrm{FcEt})_{2}\right),{ }^{\left[{ }^{[}\right]}$and bisfulvalenide diiron $(\mathrm{Fc}=\mathrm{Fc}),{ }^{[30]}$ as well as the triiodide and hexafluorophosphate salts of the corresponding radical cations, were prepared according to literature methods and checked for purity by elemental analysis (see the Supporting Information).

Analysis: UV-visible-NIR spectra were recorded on a Cary 5 UV-VisNIR spectrometer using quartz cuvettes (QX) with $d=0.5 \mathrm{~cm}$ for solution spectra or $\mathrm{KBr}$ pellets $\left(10\right.$ tons $\left.\mathrm{cm}^{-2}\right)$. Resolution was set to $1 \mathrm{~nm}$. Polystyrene films were prepared by codissolving the biferrocenyl salts and polystyrene and subsequently evaporating the solution slowly. MIR spectra were recorded with a Genesis Type I spectrometer (ATI Mattson, Madison) in the range of 400 to $6000 \mathrm{~cm}^{-1}$ with an accuracy of $2 \mathrm{~cm}^{-1}$. Substances were pressed under vacuum with $\mathrm{KBr}$ at 10 tons $\mathrm{cm}^{-2}$ or measured in solution within quartz cuvettes ( $Q X, 0.5 \mathrm{~cm}$ path length) or a $\mathrm{CaF}_{2}$ cell $(0.1 \mathrm{~cm}$ path length). Elemental analysis was performed with a Euro Vector CHNS-O-element analyzer Euro EA 3000. Samples were burned in sealed tin containers with a stream of oxygen.

In the matrix-isolation experiments, neutral $\mathrm{Fc}_{2}$ or $\mathrm{Fc}=\mathrm{Fc}$ was sublimed from a heatable tube and codeposited with a stream of argon containing $\mathrm{CH}_{2} \mathrm{Cl}_{2}(1 \mathrm{~mol} \%)$ onto a CsI plate held at $20 \mathrm{~K}$ in a closed-cycle cryostat. After deposition and recording of initial spectra, the sample was exposed to X-irradiation from a tungsten target $(40 \mathrm{kV} / 40 \mathrm{~mA})$ for $90 \mathrm{~min}$. This results in the creation of electron-hole pairs in the Ar matrix, some of which separate and move through the matrix. The electrons undergo dissociative attachment to $\mathrm{CH}_{2} \mathrm{Cl}_{2}$ whereas the holes are ultimately trans- 
ferred to the substrate, $\mathrm{Fc}_{2}$ or $\mathrm{Fc}=\mathrm{Fc}$, which is thereby ionized by electron transfer to $\mathrm{Ar}^{\cdot+} .^{[31]}$ Spectra were recorded on a Perkin-Elmer Lambda 900 spectrometer $(200-3000 \mathrm{~nm})$ and on a Bomem DA3 interferometer (IR range).

Density functional theory calculations: Spin-unrestricted DFT calculations were performed by the BP86 $6^{[32,33]}$ functional, which had proven to give satisfactory results for neutral metallocenes. ${ }^{[34]}$ In calculations using the Gaussian program package ${ }^{[35]}$ the LANL2DZ basis set was used, which implies Dunning/Huzinaga full double-zeta (D95) basis functions $^{[36]}$ on the first row and Los Alamos effective core potentials plus double-zeta (DZ) functions on all other atoms. ${ }^{[37]}$ In calculations with the Amsterdam density functional (ADF) program package ${ }^{[38]}$ a triple-zeta polarized (TZP) set of Slater-type atomic orbitals (1 set of polarization functions, frozen core) optimized for use with the zero-order regular approximation (ZORA $)^{[39]}$ to the relativistic Dirac equation was used. All structures were fully optimized with each method before performing single-point time-dependent (TD) DFT excited-state calculations. ${ }^{[40]} \mathrm{Mo}$ lecular orbitals were plotted with the ADFView facility.

\section{Results}

\section{Biferrocenyl cations}

Electronic and geometric structure: The electronic structure of $\mathrm{Fc}_{2}{ }^{+}$was modeled by DFT calculations. In the solid state the two units of the singly linked $\mathrm{Fc}_{2}{ }^{+}$are arranged in a trans configuration, as in neutral $\mathrm{Fc}_{2}$, and the cation exhibits perfect $C_{2 h}$ symmetry; ${ }^{[12]}$ so the calculations were also performed in this point group. Figure 1 shows the bond lengths

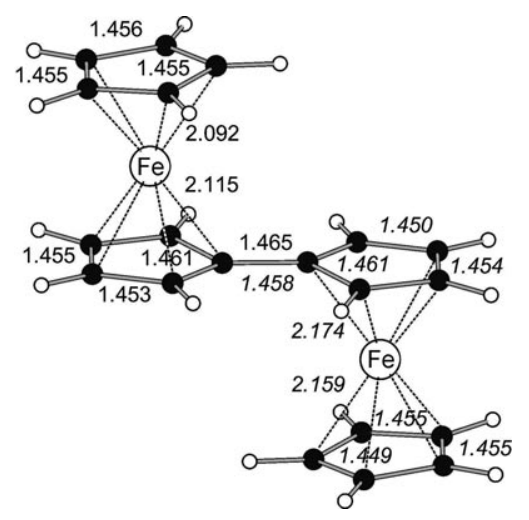

Figure 1. Structure of $\mathrm{Fc}_{2}$ and its radical cation, calculated by the BP86/ LANL2DZ method, with interatomic distances in $\AA$ indicated in normal font for the neutral dimer and in italic for the radical cation.

resulting from geometry optimizations of $\mathrm{Fc}_{2}$ and $\mathrm{Fc}_{2}{ }^{+}$by the Gaussian program (data for the geometries from the calculations with ADF can be found in the Supporting Information, Figure S1). It is seen that oxidation of the neutral dimer leads to an increase of the $\mathrm{Fe}-\mathrm{Cp}$ distances and to a slight shortening of the bond linking the two Fc moieties, whereas the bonds in the Cp rings are barely affected. These changes indicate that the MO from which ionization occurs must have some ligand character and, in particular, is slightly antibonding along the bond linking the two Fc halves.
A section of the molecular orbital scheme of $\mathrm{Fc}_{2}$ containing the relevant MOs is shown in Figure 2. The underlying coordinate system is oriented such that the $x$ axis is along the $\mathrm{C}-\mathrm{C}$ linkage and the $z$ axis is along the metal-Cp direction. The MOs of the dimer can be derived as linear combinations of those of Fc that are shown on the left-hand side of Figure 2 (Kohn-Sham orbitals in $D_{5 h}$ symmetry). On the right-hand side the resulting orbitals of $\mathrm{Fc}_{2}$ are shown. In the radical cation all orbitals of the closed-shell dimer shift and split into $\alpha$ and $\beta$ orbitals. For reasons of simplicity this is not shown in Figure 2. Moreover, only the closed-shell MOs are given as the shape of the orbitals was not found to change much upon ionization of the neutral dimer.

The metal-centered d MOs of Fc combine to give the six highest-lying MOs of $\mathrm{Fc}_{2}$. The HOMO $18 \mathrm{a}_{\mathrm{g}}$ of $\mathrm{Fc}_{2}$ (i.e., the singly occupied molecular orbital (SOMO) of $\mathrm{Fc}_{2}{ }^{+}$), corresponds to the positive combination of the $d_{x^{2}-y^{2}}$ AOs of Fc, which extends partially to the ligand and is slightly antibonding along the $\mathrm{C}-\mathrm{C}$ bond that links the two $\mathrm{Fc}$ moieties. The corresponding negative combination, $17 \mathrm{~b}_{\mathrm{u}}$, exhibits much less ligand character. The two linear combinations of the $\mathrm{d}_{x y}$ AOs of Fc are also located between these two levels. At lower energy the two $d_{z^{2}}$ combinations $17 a_{\mathrm{g}} / 16 b_{u}$ are found, which are barely split. Below these d-type levels the eight $+/-$ combinations of the Fc ligand $\pi$ orbitals $4 \mathrm{e}_{1}{ }^{\prime}$ and Fc metal-ligand bonding orbitals $3 \mathrm{e}_{1}{ }^{\prime \prime}$ are located, with $4 \mathrm{e}_{1}{ }^{\prime}$ being at higher energy than $3 \mathrm{e}_{1}{ }^{\prime \prime}$. In the dimer some hybridization between these doubly degenerate sets of orbitals occurs such that the highest-energy combination $16 \mathrm{a}_{\mathrm{g}}$ (which is antibonding with respect to the $\mathrm{C}-\mathrm{C}$ linkage) gets some bonding metal $\mathrm{d}_{x z}$ orbital contribution. The same applies to its counterpart at lower energy, $15 b_{u}$, which is slightly bonding with respect to the $\mathrm{C}-\mathrm{C}$ linkage. The two combinations in between, $10 a_{u}$ and $10 b_{g}$, have very little metal character. At even lower energy the corresponding dimer combinations of the $3 \mathrm{e}_{1}{ }^{\prime \prime} \mathrm{Fc}$ orbitals are found.

Above the occupied metal orbitals of $\mathrm{Fc}_{2}$, the four vacant MOs $18 \mathrm{~b}_{\mathrm{u}}, 19 \mathrm{a}_{\mathrm{g}}, 12 \mathrm{a}_{\mathrm{u}}$, and $12 \mathrm{~b}_{\mathrm{g}}$ are located, which are $+/-$ combinations of the Fc metal-ligand antibonding $4 \mathrm{e}_{1}{ }^{\prime \prime} \mathrm{d}_{x z}$ and $\mathrm{d}_{y z}$ orbitals, respectively. The LUMO, $18 \mathrm{~b}_{\mathrm{u}}$, has bonding character with respect to the $\mathrm{C}-\mathrm{C}$ linkage. At even higher energy, a ligand $\pi^{*}$-type orbital is found $\left(19 b_{u}\right)$, which also has some metal character and is bonding with respect to the $\mathrm{C}-\mathrm{C}$ linkage.

As is usual with DFT calculations, the valence orbital energies of neutral compounds lie about $2 \mathrm{eV}$ too high in energy, but the general pattern is in line with the photoelectron (PE) spectrum of Fc. ${ }^{[41]}$ In particular, the gap of approximately $1.5 \mathrm{eV}$ between the metal-centered $\mathrm{d}$ MOs of Fc $\left(3 \mathrm{e}_{2}{ }^{\prime}\right.$ and $5 \mathrm{a}_{1}{ }^{\prime} ; 3 \mathrm{e}_{2 \mathrm{~g}}$ and $5 \mathrm{a}_{1 \mathrm{~g}}$, respectively, in the "native" $D_{5 d}$ symmetry of Fc) and the ligand-based $4 \mathrm{e}_{1}^{\prime}$ and $3 \mathrm{e}_{1}^{\prime \prime}$ MOs $\left(4 \mathrm{e}_{1 \mathrm{u}}\right.$ and $3 \mathrm{e}_{1 \mathrm{~g}}$, respectively, in $\left.D_{5 d}\right)$ is reproduced well. Our calculations for $\mathrm{Fc}_{2}$ are also in excellent agreement with the photoelectron spectrum of this dimer, ${ }^{[41]}$ which shows a double-humped first band, the first component of which has a width of $0.56 \mathrm{eV}(0.22 \mathrm{eV}$ more than in $\mathrm{Fc})$, whereas that of the second peak is only $0.22 \mathrm{eV}(0.11 \mathrm{eV}$ more than in 


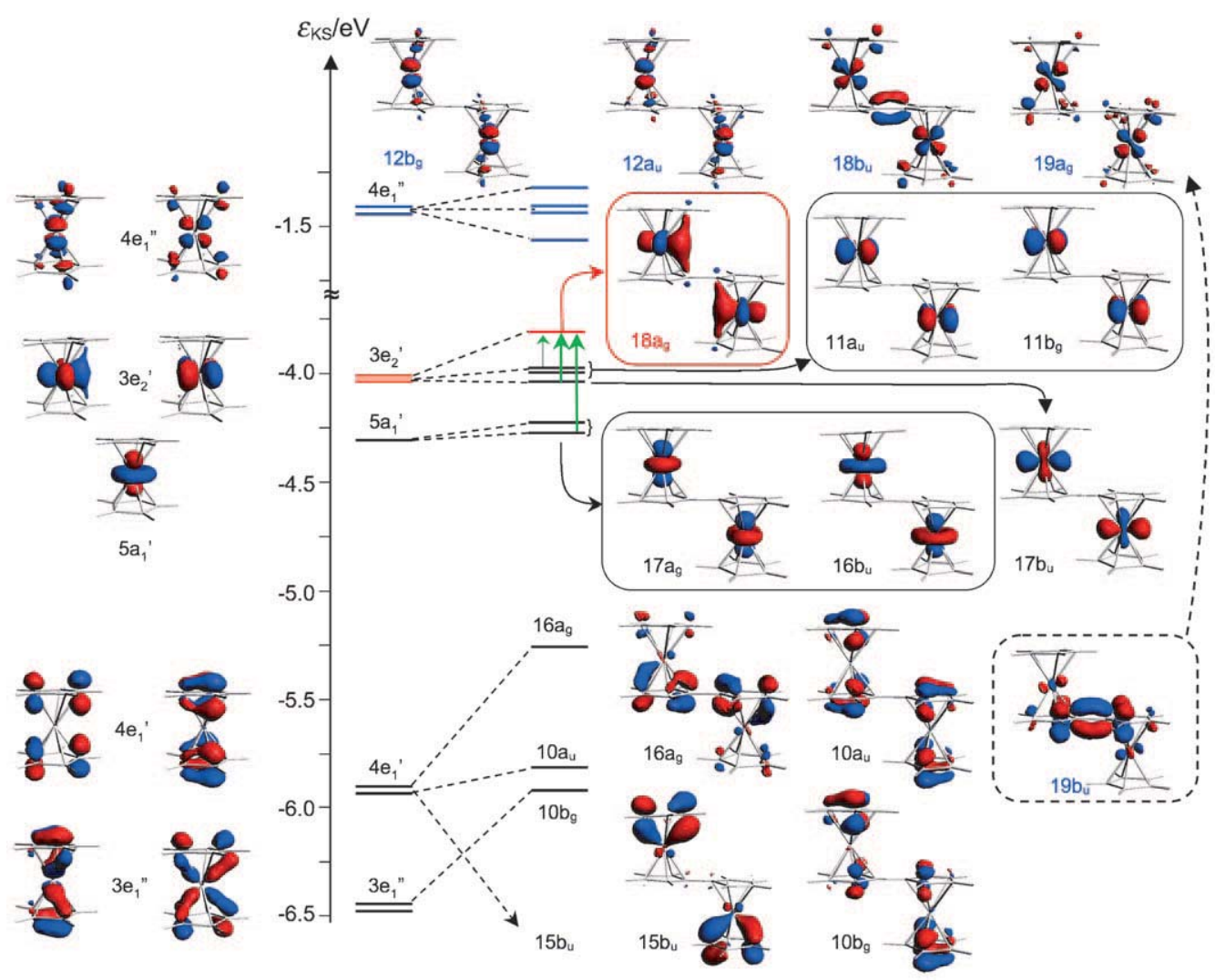

Figure 2. Kohn-Sham molecular orbitals of Fc (left-hand side) and $\mathrm{Fc}_{2}$ (right-hand side) from BP86/DZ(ZORA) calculations with the ADF program. Blue levels are virtual MOs, red levels are open shells in the radical cations, and black levels are doubly occupied MOs. Dipole-allowed IR transitions are indicated by green arrows.

Fc). According to Lichtenberger's analysis, ${ }^{[41]}$ the first, wide peak is due to the $\mathrm{d}_{x y}$ and $\mathrm{d}_{x^{2}-y^{2}}$ AOs, whereas the second, narrower peak is due to the $\mathrm{d}_{z^{2}}$ AOs of the Fc units. The present DFT calculations suggest that the width of the first peak is due mainly to a splitting of the $d_{x^{2}-y^{2}}$ levels $\left(18 \mathrm{a}_{\mathrm{g}} /\right.$ $17 b_{u}$ in $\mathrm{Fc}_{2}$ ), whereas the $\mathrm{d}_{x y}$ levels remain almost degenerate. The narrow width of the second peak is due to the small splitting of the lower-lying $\mathrm{d}_{z^{2}}$ levels $\left(17 \mathrm{a}_{\mathrm{g}} / 16 \mathrm{~b}_{\mathrm{u}}\right)$. For the latter set of MOs, our calculations predict the bonding combination $\left(17 \mathrm{a}_{\mathrm{g}}\right)$ to lie approximately $0.05 \mathrm{eV}$ higher than the antibonding one $\left(16 b_{u}\right)$, which might appear strange, but is due to subtle rehybridization among those combinations of d AOs, which become symmetry equivalent in the $C_{2 h}$ point group of $\mathrm{Fc}_{2}$.

Optical absorption spectroscopy: Figure 3 shows the optical absorption spectrum of the biferrocenyl $\left(\mathrm{Fc}_{2}{ }^{+}\right)$triiodide along with that of neutral $\mathrm{Fc}_{2}$ and the spectra of the constituents ferrocene $(\mathrm{Fc})$ and ferrocenium $\left(\mathrm{Fc}^{*+}\right)$ (as a hexafluorophosphate salt) in the range from 2500 to $50000 \mathrm{~cm}^{-1}$. In the inset the spectra are scaled up in the range below $20000 \mathrm{~cm}^{-1}$. Band positions and intensities are collected in Table 1. Importantly, $\mathrm{Fc}_{2} \mathrm{I}_{3}$ exhibits an absorption feature in the NIR/MIR region (band I) that is not present in all other systems (see Figure 3) and thus must be an intrinsic property

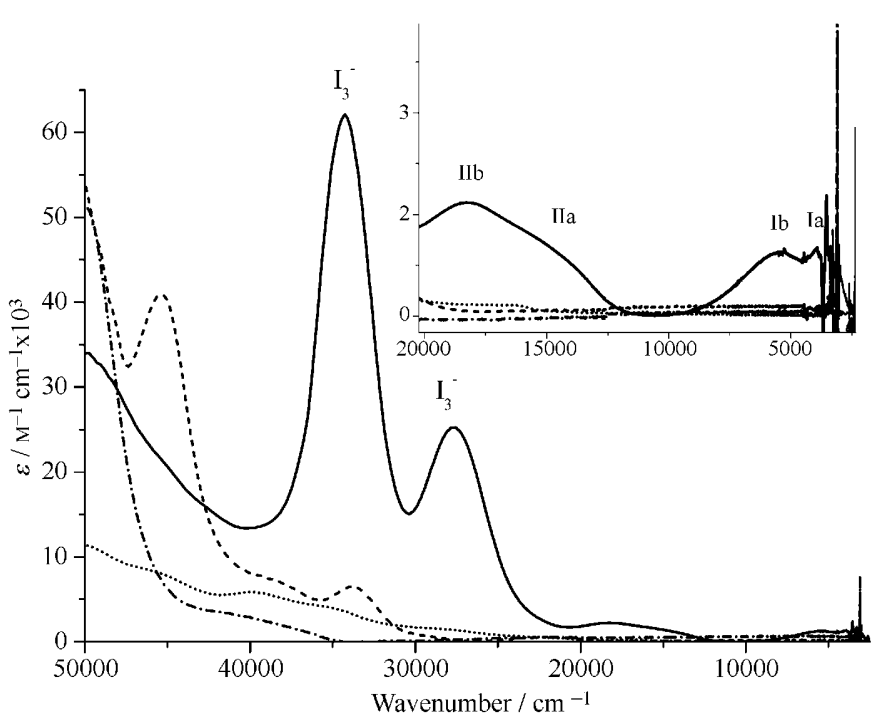

Figure 3. UV-visible-NIR-MIR spectra of biferrocenyl triiodide $\left(\mathrm{Fc}_{2} \mathrm{I}_{3}\right.$; - ) along with those of the constituent subunits ferrocene $(\mathrm{Fc} ;-\cdot \cdot-\bullet)$ and ferrocenium hexafluorophosphate $\left(\mathrm{FcPF}_{6} ; \cdots \cdot\right)$, and the neutral parent neutral compound biferrocene $\left(\mathrm{Fc}_{2} ;---\right)$ in $\left[\mathrm{D}_{3}\right]$ acetonitrile.

of $\mathrm{Fc}_{2}{ }^{\cdot+}$. The higher-energy component of this feature (Ib) had been observed previously and was attributed to an 
Table 1. Electronic absorption bands $\left(\tilde{v}\right.$ in $\left.\mathrm{cm}^{-1}\right)$ of $\mathrm{Fc}_{2}{ }^{+}$.

\begin{tabular}{|c|c|c|c|c|}
\hline \multirow{2}{*}{$\begin{array}{l}\text { Band } \\
\text { no. }\end{array}$} & \multicolumn{3}{|c|}{ Band position of sample in } & \multirow{2}{*}{$\begin{array}{l}\text { Assignment } \\
\text { of transition }^{[\mathrm{b}]}\end{array}$} \\
\hline & $\mathrm{CH}_{3} \mathrm{CN}^{[\mathrm{a}]}$ & $\mathrm{KBr}$ pellet & solid $\mathrm{Ar} / 10 \mathrm{~K}$ & \\
\hline$\overline{\mathrm{Ia}}$ & $3900(1000)$ & 3000 & 3000 & $\begin{array}{l}1^{2} \mathrm{~B}_{\mathrm{u}}\left(17 \mathrm{~b}_{\mathrm{u}} \beta \rightarrow 18 \mathrm{a}_{\mathrm{g}} \beta /\right. \\
\left.16 \mathrm{~b}_{\mathrm{u}} \beta \rightarrow 18 \mathrm{a}_{\mathrm{g}} \beta\right)\end{array}$ \\
\hline $\mathrm{Ib}$ & $5500(1000)$ & 5000 & 5000 & $\begin{array}{l}2^{2} \mathrm{~B}_{\mathrm{u}}\left(17 \mathrm{~b}_{\mathrm{u}} \beta \rightarrow 18 \mathrm{a}_{\mathrm{g}} \beta /\right. \\
\left.16 \mathrm{~b}_{\mathrm{u}} \beta \rightarrow 18 \mathrm{a}_{\mathrm{g}} \beta\right)\end{array}$ \\
\hline II a & $14100(1000)$ & 14000 & 15000 & $\begin{array}{l}4^{2} \mathrm{~B}_{\mathrm{u}}\left(15 \mathrm{~b}_{\mathrm{u}} \beta \rightarrow 18 \mathrm{a}_{\mathrm{g}} \beta\right. \\
\text { "hole transition") }\end{array}$ \\
\hline $\mathrm{II} b$ & $18200(2000)$ & 18000 & 18000 & $\begin{array}{l}7^{2} \mathrm{~B}_{\mathrm{u}}\left(18 \mathrm{a}_{\mathrm{g}} \alpha \rightarrow 18 \mathrm{~b}_{\mathrm{u}} \alpha ;\right. \\
\text { SOMO-LUMO })\end{array}$ \\
\hline III & - & - & 27000 & $\begin{array}{l}\text { ligand } \rightarrow 18 b_{u}(\mathrm{LMCT} \text {; } \\
\text { mixed with } \mathrm{LF})\end{array}$ \\
\hline
\end{tabular}

[a] $\varepsilon$ values in brackets. [b] Refer to Figure 2 and Table 5.

ICVT transition (vide supra), whereas the lower-energy component (Ia) has never been detected before. Very similar absorption features in the IR range are observed for the diethyl derivative, $(\mathrm{FcEt})_{2}{ }^{+}$(Figure 4$)$. In particular, band I

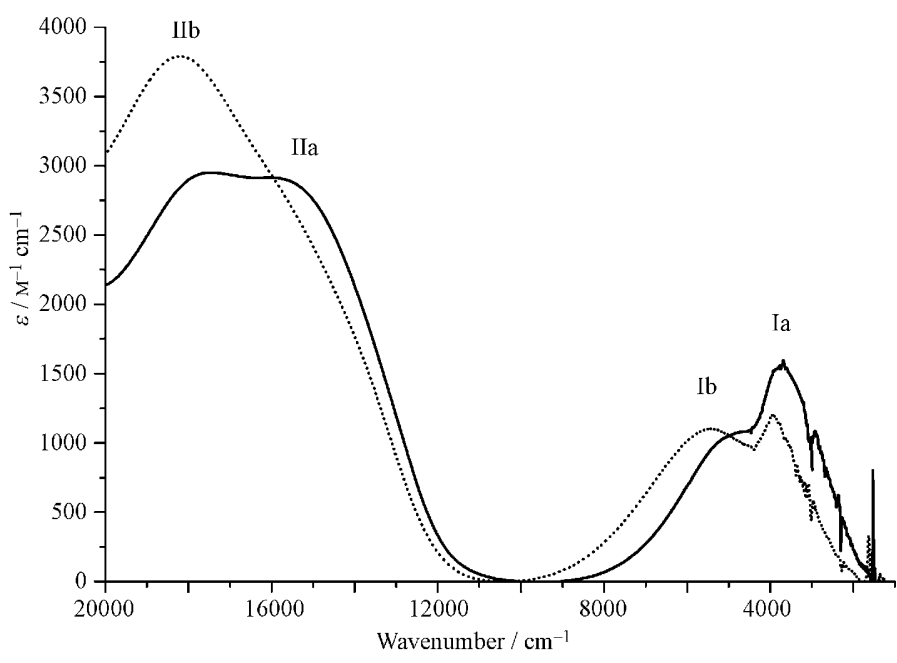

Figure 4. Low-energy bands of the $1^{\prime}, 1^{\prime \prime \prime}$-diethylbiferrocenyl triiodide $\left((\mathrm{FcEt})_{2} \mathrm{I}_{3}\right)$ in dichloromethane $(-)$ and in acetonitrile $(\cdots \cdot \cdot)$, using a $\mathrm{CaF}_{2}$ cell $(300 \mathrm{~K})$.

is again found to exhibit two maxima, one at about $3900 \mathrm{~cm}^{-1}$ (a) and the other one at $5000 \mathrm{~cm}^{-1}$ (b). When going from dichloromethane to acetonitrile, the solvent shift of the lower-energy component Ia is weaker (ca. $300 \mathrm{~cm}^{-1}$ ) than that of the higher-energy one $\left(\mathrm{Ib} ; 600 \mathrm{~cm}^{-1}\right)$. From this we conclude that the two maxima correspond to two different electronic excitations. Further solvent shifts are collected in Table 2. At higher energy $\left(\approx 17000 \mathrm{~cm}^{-1}\right)$, a second absorption feature (band II) is observed that also seems to contain at least two transitions. Upon changing to the more polar solvent this feature exhibits a marked change in appearance as well; in particular, the lower-energy component (band IIa) appears to decrease, whereas the higher-energy component (band IIb) appears to increase in intensity.

At approximately 27500 and $34000 \mathrm{~cm}^{-1}$ the spectrum of $\mathrm{Fc}_{2} \mathrm{I}_{3}$ (Figure 3) shows two intense bands which, however, are due to the triiodide counterion. Efforts to record optical
Table 2. Solvent dependence of low-energy bands $\left(\tilde{v}\right.$ in $\left.\mathrm{cm}^{-1}\right)$ for $(\mathrm{FcEt})_{2} \mathrm{I}_{3}$.

\begin{tabular}{lll}
\hline Solvent & Band Ib & Band Ia \\
\hline $\mathrm{CD}_{3} \mathrm{CN}$ & 5400 & 3900 \\
$\mathrm{CD}_{2} \mathrm{Cl}_{2}$ & 4900 & 3700 \\
{$\left[\mathrm{D}_{8}\right] \mathrm{THF}$} & 5300 & 3900 \\
$\left(\mathrm{CD}_{3}\right)_{2} \mathrm{CO}$ & 5500 & 3600 \\
$\mathrm{CD}_{3} \mathrm{Cl}$ & 4900 & 3700 \\
\hline
\end{tabular}

solution spectra of the mixed-valent dimer with counterions lacking intrinsic absorptions in the visible region (e.g., $\mathrm{PF}_{6}$ ) were unsuccessful, as in this case the IR features (bands Ia and $\mathrm{Ib}$ ) disappeared, which indicated decomposition of the $\mathrm{Fc}_{2}{ }^{++}$cation. In Figure 5 the spectra of $\mathrm{Fc}_{2} \mathrm{I}_{3}$ and $(\mathrm{FcEt})_{2} \mathrm{I}_{3}$ in

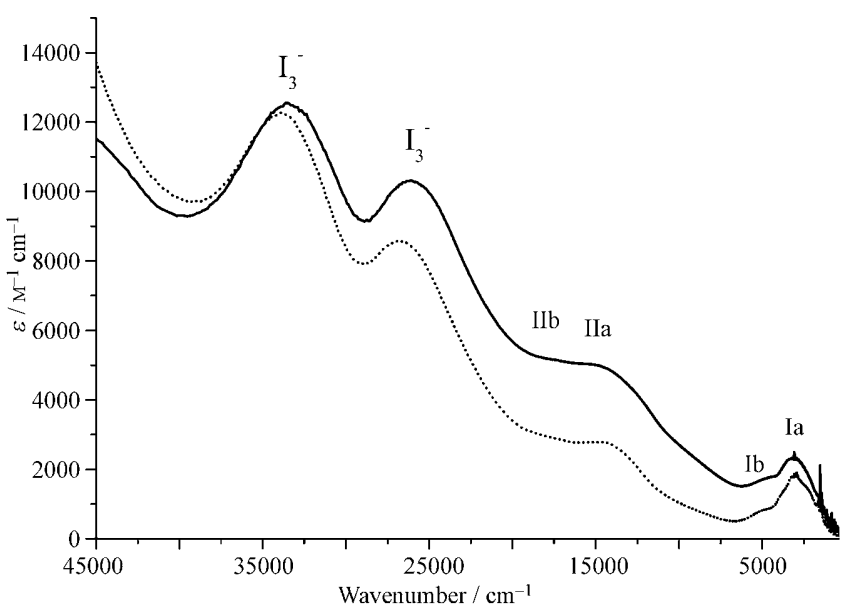

Figure 5. Visible-NIR-MIR spectra of $(\mathrm{FcEt})_{2} \mathrm{I}_{3}(\cdots \cdots)$ and $\mathrm{Fc}_{2} \mathrm{I}_{3}(-)$ in $\mathrm{KBr}$ pellets $(300 \mathrm{~K})$.

a $\mathrm{KBr}$ matrix are given. The spectra show again the pair of NIR/MIR transitions (bands Ia and Ib) at 3000 and $5000 \mathrm{~cm}^{-1}$; moreover, at 14000 and $18000 \mathrm{~cm}^{-1}$ the doublehumped absorption of band II is located. At higher energy the intense triiodide absorptions at approximately 27000 and $34000 \mathrm{~cm}^{-1}$ are observed. Interestingly, the solid-state absorption spectra seem to contain an additional absorption around $10000 \mathrm{~cm}^{-1}$, which is lacking in the solution spectra. A peak-fit analysis allows to more exactly locate this band at approximately $8750 \mathrm{~cm}^{-1}$; that is, between bands I and II (see Figure S2 in the Supporting Information). This band exists both in triiodide and hexafluorophosphate salts of $\mathrm{Fc}_{2}{ }^{+}$and $(\mathrm{FcEt})_{2}{ }^{+}$, thus eliminating the possibility of a triiodide absorption that would also lead to a broad band over the whole visible range in the solid-state spectra. The fact that this band is exclusively observed in $\mathrm{KBr}$ pellets (which contain crystallites of the compounds) but not in solution or matrix-isolation spectra (see below) leads to the conclusion that it is probably due to an intermolecular (interdimer) charge-transfer transition in the crystal. Similar observations have been made on single crystals of ferrocenium trichloroacetate; also in this case an intermolecular 
charge transfer has been invoked to explain the presence of this band. ${ }^{[42]}$

To obtain spectroscopic information about $\mathrm{Fc}_{2}{ }^{\cdot+}$ without interfering effects of the counterion, the solvent, or the crystalline environment, we turned to matrix-isolation spectroscopy. Figure 6 shows the electronic absorption spectrum of

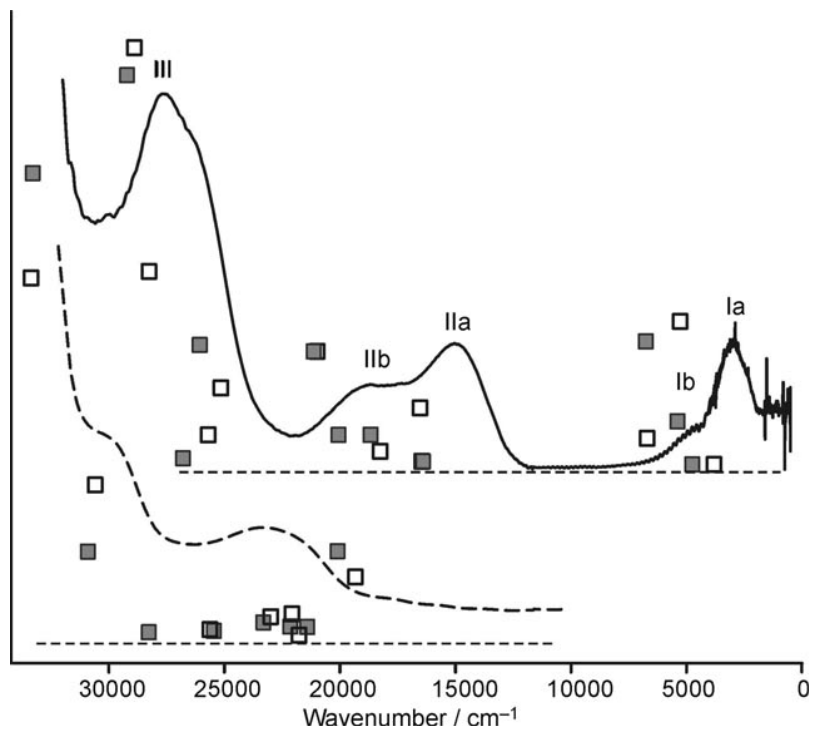

Figure 6. Electronic absorption spectra of $\mathrm{Fc}_{2}(---)$ and $\mathrm{Fc}_{2}{ }^{+}(-)$in Ar at $10 \mathrm{~K}$. The filled squares $(\square)$ denote electronic transitions predicted by TD-BP86 calculations using the TZP basis set and the ZORA method as implemented in the ADF program, whereas the open squares ( $\square$ ) refer to calculations using the LANL2DZ basis set with the Gaussian program as listed in Tables 4 (for $\mathrm{Fc}_{2}$ ) and 5 (for $\mathrm{Fc}_{2}{ }^{+}$).

$\mathrm{Fc}_{2}{ }^{++}$obtained by ionization of $\mathrm{Fc}_{2}$ in an Ar matrix at $6 \mathrm{~K}$ as well as the absorption spectrum of the parent, neutral dimer. As in these experiments the negative charge carriers are separated by one or more layers of Ar from the radical cation, the spectrum of $\mathrm{Fc}_{2}{ }^{+}$can be regarded as being due to isolated radical cations, unperturbed by any counterion, solvent (radical ions with such small electron affinities as $\mathrm{Fc}_{2}{ }^{+}$interact only very weakly with $\mathrm{Ar}$ ), or solid-state effects. Nevertheless, the spectrum shows similar bands as the above solution and solid-state spectra, except that there is now a clear gap of approximately $1 \mathrm{eV}$ between the two IR bands Ia/Ib and the double-humped band system (II) that follows in the visible range. Moreover, the Ar spectrum shows an intrinsic absorption band of the $\mathrm{Fc}_{2}{ }^{+}$radical cation (band III) at a similar position $\left(\approx 27000 \mathrm{~cm}^{-1}\right)$ at which the first triiodide absorption in the solution and solid-state absorption spectra of $\mathrm{Fc}_{2} \mathrm{I}_{3}$ is located (vide supra). Above $32000 \mathrm{~cm}^{-1}$ the spectrum in $\mathrm{Ar}$ is masked by strong absorptions of $\mathrm{Fc}_{2}$ (only $10-15 \%$ of which are converted to the radical cation in this experiment).

Importantly, the spectrum of the neutral dimer is very different from that of the radical cation, showing an absorption at approximately $23000 \mathrm{~cm}^{-1}$ and a shoulder at $30000 \mathrm{~cm}^{-1}$. (Table 3). The solution spectrum of $\mathrm{Fc}_{2}$ given in Figure 3
Table 3. Electronic absorption bands $\left(\tilde{v}\right.$ in $\left.\mathrm{cm}^{-1}\right)$ of $\mathrm{Fc}_{2}$.

\begin{tabular}{llr}
\hline \multicolumn{2}{c}{ Band position of sample in } \\
$\mathrm{CH}_{3} \mathrm{CN}^{[\mathrm{a}]}$
\end{tabular}$\quad \begin{aligned} & \text { solid Ar/10 K } \\
& 23000(500)\end{aligned}$

[a] $\varepsilon$ values in brackets. [b] Refer to Figure 2 and Table 4. [c] sh. $=$ shoulder. [d] In ADF mixed with $18 \mathrm{a}_{\mathrm{g}}(\mathrm{HOMO}) \rightarrow 19 \mathrm{~b}_{\mathrm{u}}$, MLCT.

further indicates the presence of an intense absorption band at $33000 \mathrm{~cm}^{-1}$. We also recorded the IR spectra of $\mathrm{Fc}_{2}$ and its radical cation (see Figure S3 in the Supporting Information), and both are in good agreement with the calculated spectra for the two species (see the Supporting Information).

A comparison of the spectra of $\mathrm{Fc}_{2}{ }^{+}$in solution $\left(\mathrm{CH}_{3} \mathrm{CN}\right), \mathrm{KBr}$, and $\mathrm{Ar}$ is given in Figure 7. As clearly shown by this figure, qualitatively similar spectra are obtained. However, there are conspicuous differences in band positions and intensities. In particular, it is evident that the IR bands I a and Ib are markedly shifted to higher energies in the solution spectrum, which can be attributed to solvent effects. Moreover, the intensity distribution of the two components of band I in the Ar spectrum is reversed in solution: whereas in the former case the high-energy transition is much less intense than the transition at lower energy, the high-energy transition becomes more intense than the lowenergy transition in solution. Similar changes are observed in the double-humped band at $18000 \mathrm{~cm}^{-1}$ (band II) when going from the Ar matrix to the solution spectrum. The spectral differences at higher energy are due to the fact that matrix-isolation data show an intrinsic absorption band of the $\mathrm{Fc}_{2}{ }^{+}$radical cation (band III) at a similar position (

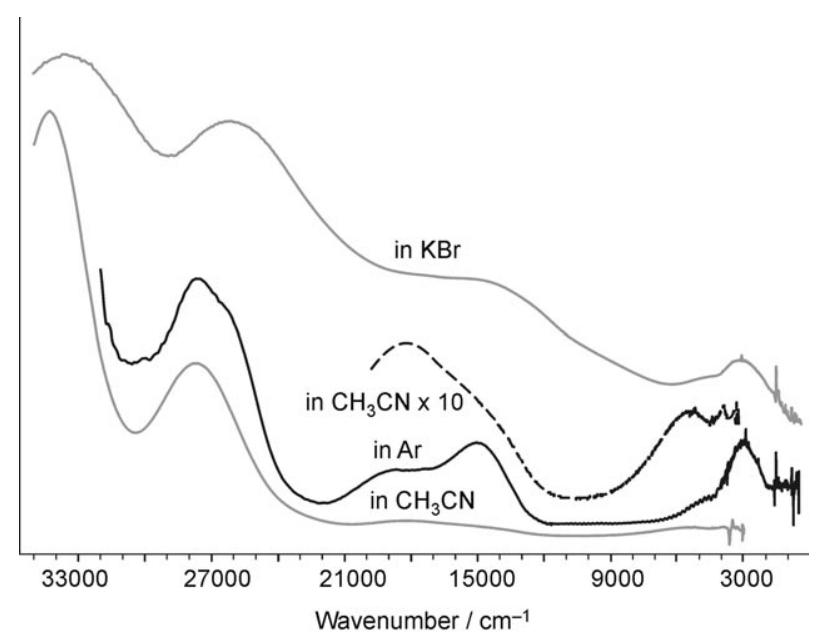

Figure 7. Comparison of optical absorption spectra of $\mathrm{Fc}_{2}{ }^{+}$in $\mathrm{KBr}$, $\mathrm{CH}_{3} \mathrm{CN}$ (also multiplied by 10 for clarity), and an Ar matrix. 
$\approx 27000 \mathrm{~cm}^{-1}$ ) as the first triiodide absorption in the solution and solid-state absorption spectra of $\mathrm{Fc}_{2} \mathrm{I}_{3}$ (vide supra).

Upon lowering the temperature from 300 to $10 \mathrm{~K}$, the band shapes change in the spectra taken in $\mathrm{KBr}$ pellets, but the overall change in intensity is low. In contrast, the absorption spectrum recorded in a polystyrene matrix is almost temperature independent (data not shown); as a matter of fact this spectrum is more related to that taken in dichloromethane (cf. Figure 4). The temperature dependence of the solid-state spectra is considered in more detail in a forthcoming paper.

TD-DFT calculations and band assignments: To assign the electronic absorption bands of $\mathrm{Fc}_{2}$ and $\mathrm{Fc}_{2}{ }^{-}$, TD-DFT calculations were performed. As before, two different basis sets and programs were employed (ADF, G03; cf. the Experimental Section). The results of the calculations are collected in Tables 4 and 5 for $\mathrm{Fc}_{2}$ and $\mathrm{Fc}_{2}{ }^{+}$, respectively. Moreover, the positions and intensities of the electronic transitions calculated by means of the ADF and Gaussian 03 programs are indicated schematically in Figure 6. The resulting assignments of experimentally observed bands are included in Tables 1 and 3 .

Table 4. Electronic transitions $\left(\tilde{v}\right.$ in $\left.\mathrm{cm}^{-1}\right)$ of $\mathrm{Fc}_{2}$ calculated by the TDBP86 method.

\begin{tabular}{|c|c|c|c|c|c|}
\hline \multirow[t]{2}{*}{$\overline{\text { States }}$} & \multicolumn{2}{|c|}{$\mathrm{ADF}^{[\mathrm{a}]}$} & \multicolumn{2}{|c|}{ Gaussian $^{[\mathrm{b}]}$} & \multirow{2}{*}{$\begin{array}{l}\text { Configurational } \\
\text { composition }^{[\mathrm{c}]}\end{array}$} \\
\hline & $\tilde{v}\left[\mathrm{~cm}^{-1}\right]$ & $f$ & $\tilde{v}\left[\mathrm{~cm}^{-1}\right]$ & $f$ & \\
\hline $1^{1} \mathrm{~B}_{\mathrm{u}}$ & 20168 & 0.0443 & 19361 & 0.0320 & $\begin{array}{l}18 \mathrm{a}_{\mathrm{g}} \rightarrow 18 \mathrm{~b}_{\mathrm{u}}(0.86 / 0.61) \\
11 \mathrm{a}_{\mathrm{u}} \rightarrow 12 \mathrm{~b}_{\mathrm{g}}(-/ 0.25)\end{array}$ \\
\hline $2^{1} B_{u}$ & 21458 & 0.0048 & 21761 & 0.0004 & $\begin{array}{l}11 \mathrm{~b}_{\mathrm{g}} \rightarrow 12 \mathrm{a}_{\mathrm{u}}(0.56 / 0.60) \\
11 \mathrm{a}_{\mathrm{u}} \rightarrow 12 \mathrm{~b}_{\mathrm{g}}(0.42 / 0.39)\end{array}$ \\
\hline $3^{1} \mathrm{~B}_{\mathrm{u}}$ & 22184 & 0.0036 & 22104 & 0.0108 & $\begin{array}{l}17 \mathrm{a}_{\mathrm{g}} \rightarrow 18 \mathrm{~b}_{\mathrm{u}}(0.89 / 0.52) \\
11 \mathrm{a}_{\mathrm{u}} \rightarrow 12 \mathrm{~b}_{\mathrm{g}}(-/ 0.40)\end{array}$ \\
\hline $4^{1} \mathrm{~B}_{\mathrm{u}}$ & 23365 & 0.0067 & 22991 & 0.0103 & $\begin{array}{l}17 \mathrm{~b}_{\mathrm{u}} \rightarrow 19 \mathrm{a}_{\mathrm{g}}(0.48 / 0.52) \\
17 \mathrm{a}_{\mathrm{g}} \rightarrow 18 \mathrm{~b}_{\mathrm{u}}(-/ 0.37) \\
11 \mathrm{a}_{\mathrm{u}} \rightarrow 12 \mathrm{~b}_{\mathrm{g}}(0.26 / 0.21) \\
11 \mathrm{~b}_{\mathrm{g}} \rightarrow 12 \mathrm{a}_{\mathrm{u}}(0.22 /-)\end{array}$ \\
\hline $5^{1} \mathrm{~B}_{\mathrm{u}}$ & 25503 & 0.0026 & 25653 & 0.0038 & $\begin{array}{l}16 \mathrm{~b}_{\mathrm{u}} \rightarrow 19 \mathrm{a}_{\mathrm{g}}(0.70 / 0.56) \\
17 \mathrm{~b}_{\mathrm{u}} \rightarrow 19 \mathrm{a}_{\mathrm{g}}(0.20 / 0.30) \\
17 \mathrm{a}_{\mathrm{g}} \rightarrow 18 \mathrm{~b}_{\mathrm{u}}(-/ 0.30)\end{array}$ \\
\hline $6^{1} \mathrm{~B}_{\mathrm{u}}$ & 28405 & 0.0020 & 28396 & 0.0020 & $\begin{array}{l}16 \mathrm{~b}_{\mathrm{u}} \rightarrow 19 \mathrm{a}_{\mathrm{g}}(0.25 / 0.41) \\
17 \mathrm{~b}_{\mathrm{u}} \rightarrow 19 \mathrm{a}_{\mathrm{g}}(0.26 / 0.31)\end{array}$ \\
\hline $7^{1} \mathrm{~B}_{\mathrm{u}}$ & 30968 & 0.0442 & 30655 & 0.0839 & $\begin{array}{l}16 \mathrm{a}_{\mathrm{g}} \rightarrow 18 \mathrm{~b}_{\mathrm{u}}(0.65 / 0.59) \\
18 \mathrm{a}_{\mathrm{g}} \rightarrow 19 \mathrm{~b}_{\mathrm{u}}(0.65 /-)\end{array}$ \\
\hline $8^{1} \mathrm{~B}_{\mathrm{u}}$ & 33355 & 0.2421 & 33409 & 0.1970 & $\begin{array}{l}18 \mathrm{a}_{\mathrm{g}} \rightarrow 19 \mathrm{~b}_{\mathrm{u}}(0.56 / 0.56) \\
16 \mathrm{a}_{\mathrm{g}} \rightarrow 18 \mathrm{~b}_{\mathrm{u}}(0.25 / 0.26)\end{array}$ \\
\hline
\end{tabular}

[a] BP86/TZP/ZORA. [b] BP86/LANL2DZ. [c] In terms of the MOs depicted in Figure 2. The coefficients of the CIS-type wave function are from the (ADF/Gaussian) calculations, respectively.

For neutral $\mathrm{Fc}_{2}$ (cf. spectra in Figures 3 and 6) the assignments are fairly clear cut: the band at approximately $23000 \mathrm{~cm}^{-1}$ most probably is associated with the $18 \mathrm{a}_{\mathrm{g}} \rightarrow 18 \mathrm{~b}_{\mathrm{u}}$ excitation $\left(1^{1} \mathrm{~B}_{\mathrm{u}}\right.$ state), which corresponds to the HOMOLUMO transition $\left(\mathrm{d}_{x^{2}-y^{2}} \rightarrow \mathrm{d}_{x z}\right)$ in this dimer that is predicted a bit too low in energy by both methods (Table 4). The shoulder at approximately $30000 \mathrm{~cm}^{-1}$, which also is visible
Table 5. Electronic transitions $\left(\tilde{v}\right.$ in $\left.\mathrm{cm}^{-1}\right)$ of $\mathrm{Fc}_{2}{ }^{+}$calculated by the TDBP86 method.

\begin{tabular}{|c|c|c|c|c|c|}
\hline \multirow[t]{2}{*}{ States } & \multicolumn{2}{|c|}{$\mathrm{ADF}^{[\mathrm{a}]}$} & \multicolumn{2}{|c|}{ Gaussian $^{[b]}$} & \multirow{2}{*}{$\begin{array}{l}\text { Configurational } \\
\text { composition }^{[\mathrm{c}]}\end{array}$} \\
\hline & $\tilde{v}\left[\mathrm{~cm}^{-1}\right]$ & $f$ & $\tilde{v}\left[\mathrm{~cm}^{-1}\right]$ & $f$ & \\
\hline $1^{2} \mathrm{~A}_{\mathrm{u}}$ & 4760 & $<10^{-4}$ & 3791 & $<10^{-4}$ & $11 \mathrm{a}_{\mathrm{u}} \beta \rightarrow 18 \mathrm{a}_{\mathrm{g}} \beta(-/ 1.03)$ \\
\hline $1^{2} \mathrm{~B}_{\mathrm{u}}$ & 5405 & 0.013 & 5258 & 0.043 & $\begin{array}{l}17 \mathrm{~b}_{\mathrm{u}} \beta \rightarrow 18 \mathrm{a}_{\mathrm{g}} \beta(0.52 / 0.66) \\
16 \mathrm{~b}_{\mathrm{u}} \beta \rightarrow 18 \mathrm{a}_{\mathrm{g}} \beta(0.47 / 0.27)\end{array}$ \\
\hline $2^{2} \mathrm{~B}_{\mathrm{u}}$ & 6775 & 0.037 & 6678 & 0.008 & $\begin{array}{l}17 \mathrm{~b}_{\mathrm{u}} \beta \rightarrow 18 \mathrm{a}_{\mathrm{g}} \beta(0.45 / 0.13) \\
16 \mathrm{~b}_{\mathrm{u}} \beta \rightarrow 18 \mathrm{a}_{\mathrm{g}} \beta(0.52 / 1.01)\end{array}$ \\
\hline $2^{2} \mathrm{~A}_{\mathrm{u}}$ & 16375 & 0.001 & 16428 & 0.001 & $11 \mathrm{a}_{\mathrm{u}} \beta \rightarrow 18 \mathrm{a}_{\mathrm{g}} \beta(0.99 / 0.90)$ \\
\hline $4^{2} \mathrm{~B}_{\mathrm{u}}$ & 18635 & 0.009 & 16495 & 0.017 & $\begin{array}{l}15 \mathrm{~b}_{\mathrm{u}} \beta \rightarrow 18 \mathrm{a}_{\mathrm{g}} \beta(0.94 / 0.42) \\
17 \mathrm{a}_{\mathrm{g}} \beta \rightarrow 18 \mathrm{~b}_{\mathrm{u}} \beta(0.02 / 0.37) \\
17 \mathrm{a}_{\mathrm{g}} \alpha \rightarrow 18 \mathrm{~b}_{\mathrm{u}} \alpha(0.02 / 0.21) \\
17 \mathrm{~b}_{\mathrm{u}} \beta \rightarrow 19 \mathrm{a}_{\mathrm{g}} \beta(0.01 / 0.55)\end{array}$ \\
\hline $5^{2} \mathrm{~B}_{\mathrm{u}}$ & 20005 & 0.009 & 18237 & 0.004 & $\begin{array}{l}17 \mathrm{~b}_{\mathrm{u}} \beta \rightarrow 19 \mathrm{a}_{\mathrm{g}} \beta(0.61 / 0.34) \\
17 \mathrm{a}_{\mathrm{g}} \alpha \rightarrow 18 \mathrm{~b}_{\mathrm{u}} \alpha(0.17 / \\
<0.01) \\
15 \mathrm{~b}_{\mathrm{u}} \beta \rightarrow 18 \mathrm{a}_{\mathrm{g}} \beta(0.04 / 0.56)\end{array}$ \\
\hline $7^{2} \mathrm{~B}_{\mathrm{u}}$ & 21092 & 0.034 & 20945 & 0.034 & $\begin{array}{l}18 \mathrm{a}_{\mathrm{g}} \alpha \rightarrow 18 \mathrm{~b}_{\mathrm{u}} \alpha(0.44 / 0.57) \\
17 \mathrm{a}_{\mathrm{g}} \beta \rightarrow 18 \mathrm{~b}_{\mathrm{u}} \beta(0.36 / 0.57)\end{array}$ \\
\hline $\begin{array}{l}12 / \\
13^{2} \mathrm{~B}_{\mathrm{u}}\end{array}$ & 25994 & 0.036 & 25109 & 0.023 & $\begin{array}{l}16 \mathrm{a}_{\mathrm{g}} \alpha \rightarrow 18 \mathrm{~b}_{\mathrm{u}} \alpha(0.51 / 0.66) \\
16 \mathrm{a}_{\mathrm{g}} \beta \rightarrow 18 \mathrm{~b}_{\mathrm{u}} \beta(0.22 / 0.43) \\
16 \mathrm{~b}_{\mathrm{u}} \alpha \rightarrow 19 \mathrm{a}_{\mathrm{g}} \alpha(-/ 0.52) \\
14 \mathrm{~b}_{\mathrm{u}} \beta \rightarrow 18 \mathrm{a}_{\mathrm{g}} \beta(0.22 /-)\end{array}$ \\
\hline $\begin{array}{l}14 / \\
15^{2} \mathrm{~B}_{\mathrm{u}}\end{array}$ & 26719 & 0.002 & 25653 & 0.009 & $\begin{array}{l}16 \mathrm{~b}_{\mathrm{u}} \alpha \rightarrow 9 \mathrm{a}_{\mathrm{g}} \alpha(0.70 /-) \\
14 \mathrm{~b}_{\mathrm{u}} \beta \rightarrow 18 \mathrm{a}_{\mathrm{g}} \beta(-/ 0.70)\end{array}$ \\
\hline $\begin{array}{l}14 / \\
15^{2} \mathrm{~B}_{\mathrm{u}}\end{array}$ & & & 28216 & 0.058 & $\begin{array}{l}15 \mathrm{a}_{\mathrm{g}} \alpha \rightarrow 18 \mathrm{~b}_{\mathrm{u}} \alpha(-/ 0.57) \\
15 \mathrm{a}_{\mathrm{g}} \beta \rightarrow 18 \mathrm{~b}_{\mathrm{u}} \beta(-/ 0.41)\end{array}$ \\
\hline $16^{2} \mathrm{~B}_{\mathrm{u}}$ & 29126 & 0.117 & 28859 & 0.125 & $\begin{array}{l}16 \mathrm{a}_{\mathrm{g}} \beta \rightarrow 18 \mathrm{~b}_{\mathrm{u}} \beta(0.40 / 0.67) \\
15 \mathrm{a}_{\mathrm{g}} \beta \rightarrow 18 \mathrm{~b}_{\mathrm{u}} \beta(-/ 0.33) \\
10 \mathrm{a}_{\mathrm{u}} \alpha \rightarrow 12 \mathrm{~b}_{\mathrm{g}} \alpha(-/ 0.31)\end{array}$ \\
\hline
\end{tabular}

[a] BP86/TZP/ZORA. [b] BP86/LANL2DZ. [c] In terms of the MOs depicted in Figure 2. The coefficients of the CIS-type wave functions are from the (ADF/Gaussian) calculations, respectively.

in the solution spectrum of $\mathrm{Fc}_{2}$ is probably associated with the transition to the $7^{1} \mathrm{~B}_{1 \mathrm{u}}$ state, which corresponds to the $16 \mathrm{a}_{\mathrm{g}} \rightarrow 18 \mathrm{~b}_{\mathrm{u}}(\mathrm{LUMO})$ excitation, a ligand $\rightarrow$ metal $\quad \mathrm{CT}$ (LMCT) transition. In the ADF calculations this excitation is mixed with the $18 \mathrm{a}_{\mathrm{g}}(\mathrm{HOMO}) \rightarrow 19 \mathrm{~b}_{\mathrm{u}}$ transition, a metal $\rightarrow$ ligand CT (MLCT) transition. The intense band at approximately $33000 \mathrm{~cm}^{-1}$ corresponds to the $8^{1} \mathrm{~B}_{\mathrm{u}}$ state, and is associated with the same charge-transfer transitions, but this time with a preponderance of the $18 \mathrm{a}_{\mathrm{g}} \rightarrow 19 \mathrm{~b}_{\mathrm{u}}$ LMCT transition. Between the $1^{1} \mathrm{~B}_{\mathrm{u}}$ and the $7^{1} \mathrm{~B}_{\mathrm{u}}$ state, five ligand-field excited states $\left(2^{1} \mathrm{~B}_{\mathrm{u}}\right.$ to $\left.6^{1} \mathrm{~B}_{\mathrm{u}}\right)$ are located that are associated with transitions of $\mathrm{d}_{x^{2}-y^{2}} \rightarrow \mathrm{d}_{x z}, \mathrm{~d}_{x y} \rightarrow \mathrm{d}_{y z}$, and $\mathrm{d}_{z^{2}} \rightarrow \mathrm{d}_{x z}$ character. The corresponding intensities, however, are predicted to be weak by both methods and are therefore not expected to give rise to distinct bands.

Table 5 shows the results of TD-DFT calculations on $\mathrm{Fc}_{2}{ }^{+}$. We had to include over 100 roots in these calculations to reach $30000 \mathrm{~cm}^{-1}$ (most of the transitions are not listed in Table 5 because they are either dipole forbidden or carry vanishingly small oscillator strengths), so we refrained from carrying out calculations of higher-lying excited states, because transitions to such states could not be observed. In line with expectations from the MO scheme in Figure 2, these calculations predict three dipole-allowed transitions to occur in the low-energy range (below $7000 \mathrm{~cm}^{-1}$ ), all of which involve mainly excitation of an electron from one of 
the metal-centered MOs to the $18 \mathrm{a}_{\mathrm{g}}$ SOMO. The first of these transitions leads to a ${ }^{2} \mathrm{~A}_{\mathrm{u}}$ excited state, but because it is polarized perpendicular to the metal-metal axis, its oscillator strength is too weak for it to be observed experimentally under our conditions (it corresponds to the low-lying ${ }^{2} \mathrm{~A}_{1 \mathrm{~g}} \rightarrow{ }^{2} \mathrm{E}_{2 \mathrm{~g}}$ IR transition in $\mathrm{Fc}^{\cdot+}$, which has also never been observed experimentally. ${ }^{[42]}$ The following two transitions to ${ }^{2} \mathrm{~B}_{\mathrm{u}}$ excited states that lie $0.65-0.85 \mathrm{eV}$ above the ground state are more intense, with one being three to five times stronger than the other. The two basis sets (and the associated modeling of the Fe core AOs) that we have employed in our study give slightly different quantitative predictions with regard to these states (the ordering of the two $B_{u}$ states even appears to change between the two models), but the calculations leave little doubt that the two mid-IR bands Ia and $\mathrm{Ib}$ that we have observed are due to these two ${ }^{2} \mathrm{~A}_{\mathrm{g}} \rightarrow{ }^{2} \mathrm{~B}_{\mathrm{u}}$ transitions, one of which ( $\mathrm{Ib}$ ) shows more solvatochromism than the other (Ia).

Following these essentially metal-centered transitions there is a gap of over $1 \mathrm{eV}$ in the calculated spectrum, again in agreement with the $\mathrm{PE}$ spectrum of $\mathrm{Fc}_{2}$ and the optical spectrum obtained in an Ar matrix (which is free of manifestations due to interactions of $\mathrm{Fc}_{2}{ }^{+}$with itself, its counterions, or solvents; see above). After that gap we find another very weak transition to an $A_{u}$ state, followed by three more intense transitions to $B_{u}$ excited states. These involve excitations of electrons from doubly occupied metal- $\left(17 \mathrm{~b}_{\mathrm{u}}, 17 \mathrm{a}_{\mathrm{g}}\right)$ or ligand-based MOs $\left(15 \mathrm{~b}_{\mathrm{u}}\right)$ to the $18 \mathrm{a}_{\mathrm{g}}$ SOMO and/or to virtual metal/ligand-type MOs $\left(18 \mathrm{~b}_{\mathrm{u}}, 19 \mathrm{a}_{\mathrm{g}}\right)$. The largest amplitudes within these excitations are associated with the transition from the ligand-type orbital $15 b_{u}$ to the SOMO (corresponding to the "hole transition" of $\mathrm{Fc}^{+}$) and the $\mathrm{SOMO} \rightarrow$ LUMO transition $\left(18 \mathrm{a}_{\mathrm{g}} \alpha \rightarrow 18 \mathrm{~b}_{\mathrm{u}} \alpha\right)$, but the weighting of the excitations differs considerably between the two DFT methods. However, both of them position the latter transition at approximately $21000 \mathrm{~cm}^{-1}$, about $1000 \mathrm{~cm}^{-1}$ higher in energy than the (comparatively "pure") $\mathrm{HOMO} \rightarrow$ LUMO transition in neutral $\mathrm{Fc}_{2}$ (vide supra). The hole transition is located by ADF at approximately $18600 \mathrm{~cm}^{-1}$, $2400 \mathrm{~cm}^{-1}$ higher than its counterpart in monomeric $\mathrm{Fc}^{+}$ $\left(16200 \mathrm{~cm}^{-1}\right)$. G03 distributes the $15 \mathrm{~b}_{\mathrm{u}} \rightarrow 18 \mathrm{a}_{\mathrm{g}}$ excitation over two bands, one at $16500 \mathrm{~cm}^{-1}$ and one at $18200 \mathrm{~cm}^{-1}$ (the intensity of the first one being, however, much higher than that of the second one).

In summary, TD-DFT predicts several transitions in the range of 7000 to $22000 \mathrm{~cm}^{-1}$, the most intense of which is associated with the SOMO $\rightarrow$ LUMO excitation. These transitions must be associated with the double-humped band II, but the calculations do not allow an unambiguous assignment. Based on the fact that the calculations predict the SOMO-LUMO transition of $\mathrm{Fc}_{2}{ }^{-+}$to be close in energy to the HOMO-LUMO transition of $\mathrm{Fc}_{2}$ (i.e., at $\approx 20000 \mathrm{~cm}^{-1}$ ) and the hole transitions are predicted by both methods at lower energy, we are tempted to assign the lower-energy component of band II (II a) to excitations of the latter type (cf. Table 1). This would also straightforwardly account for the fact that the visible part of the absorption spectrum of
$\mathrm{Fc}_{2}{ }^{\cdot+}$ extends to much lower energy $(\approx 1 \mathrm{eV})$ than that of $\mathrm{Fc}_{2}$, due to the existence of a hole in the valence shell. In comparison with the experimental spectrum all predictions appear $1000-2000 \mathrm{~cm}^{-1}$ too high in energy, which also applies to the two IR transitions Ia and Ib.

Between 22000 and $30000 \mathrm{~cm}^{-1}$ TD-DFT predicts excitations with nonvanishing intensities to three to four additional ${ }^{2} B_{u}$ excited states. These involve excitations from ligandtype orbitals to the metal-type LUMO $\left(18 b_{u}\right)$; namely, LMCT transitions, mixed with ligand-field (LF) transitions $\left(16 \mathrm{~b}_{\mathrm{u}} \rightarrow 19 \mathrm{a}_{\mathrm{g}}\right)$, and probably are associated with band III and its shoulder in the Ar matrix spectrum of $\mathrm{Fc}_{2}{ }^{+}$. In contrast to neutral $\mathrm{Fc}_{2}$, no mixing with MLCT transitions is predicted for this part of the spectrum, probably as a consequence of the higher charge of the iron centers.

\section{Bisfulvalenide diiron cation}

Geometric and electronic structure: Although closely related to the singly bridged dimers $\mathbf{1}$ and $\mathbf{2}$, the doubly bridged bisfulvalenide diiron cation, $\mathrm{Fc}=\mathrm{Fc}^{*+}(\mathbf{3})$, differs from the former systems insofar as oxidation of the doubly bridged biferrocenylene has a larger impact on the molecular structure, especially the metal-metal distance. Whereas in the neutral compound this distance is $3.98 \AA$, it decreases in the hexafluorophosphate ${ }^{[43]}$ salt of the monocation to $3.68 \AA$ and in the picrate hemihydroquinone ${ }^{[44]}$ system to $3.63 \AA$. These findings indicate a stronger metal-metal interaction than that present in $\mathrm{Fc}_{2}{ }^{-+}$, which also is reflected by the fact that the planes of the $\mathrm{Cp}$ rings of each fulvalenide ligand are rotated by about $10^{\circ}$ around an axis perpendicular to the $\mathrm{C}-\mathrm{C}$ linkage such that the cross section of the $\mathrm{Fc}=\mathrm{Fc}^{\circ+}$ cation through the centers of the fulvalenide ligands and the $\mathrm{Fe}$ atoms is distorted from rectangular to ellipsoidal. ${ }^{[44]}$ All these features are clearly borne out by the DFT calculations (see Figure 8 for the G03 geometries; the ADF geometries are shown in Figure $\mathrm{S} 4$ in the Supporting Information). The coordinate system is chosen such that the $x$ axis is along the metal-metal direction and the $z$ axis along the metal-Cp direction.

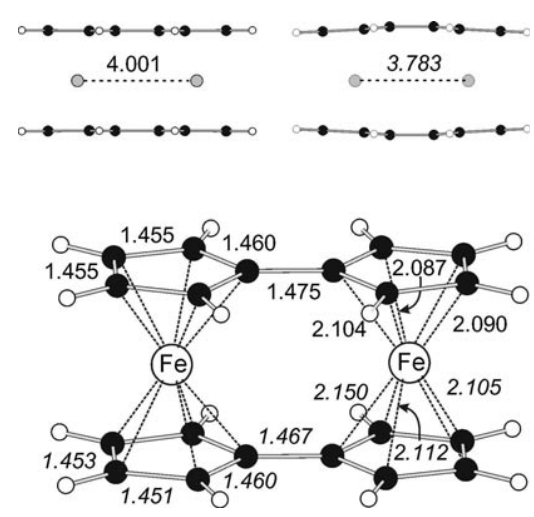

Figure 8. Structure of $\mathrm{Fc}=\mathrm{Fc}$ and its radical cation, calculated by the BP86/LANL2DZ method, with interatomic distances [̊̊] indicated in normal font for the neutral dimer and in italic for the radical cation. 
A section of the molecular orbital scheme of $\mathrm{Fc}=\mathrm{Fc}$ containing the relevant MOs (calculated at the optimized structure of $\mathrm{Fc}=\mathrm{Fc}^{\circ}$ ) is shown in Figure 9. Again dimer MOs can be derived as linear combinations of those of $\mathrm{Fc}$, which are shown on the left-hand side of Figure 2 above. In particular, the metal-centered MOs of Fc combine to give the six highest occupied MOs of $\mathrm{Fc}=\mathrm{Fc}$. The $\mathrm{HOMO} 9 \mathrm{~b}_{2 \mathrm{u}}$ (i.e., the singly occupied $\mathrm{MO}$ of $\mathrm{Fc}=\mathrm{Fc}^{\cdot+}$ ) corresponds to the negative combination of the $d_{x^{2}-y^{2}}$ AOs of $\mathrm{Fc}$, which again extends somewhat to the ligand and is slightly antibonding along the $\mathrm{C}-\mathrm{C}$ bonds linking the two Fc moieties, which explains why their distance shrinks on ionization. The corresponding bonding combination is the $10 \mathrm{a}_{\mathrm{g}} \mathrm{MO}$, which exhibits almost no ligand character. Between these two levels we find the four closely spaced metal-centered MOs that arise through linear combination of the $\mathrm{d}_{z^{2}}$ and the $\mathrm{d}_{x y}$ AOs of Fc. At

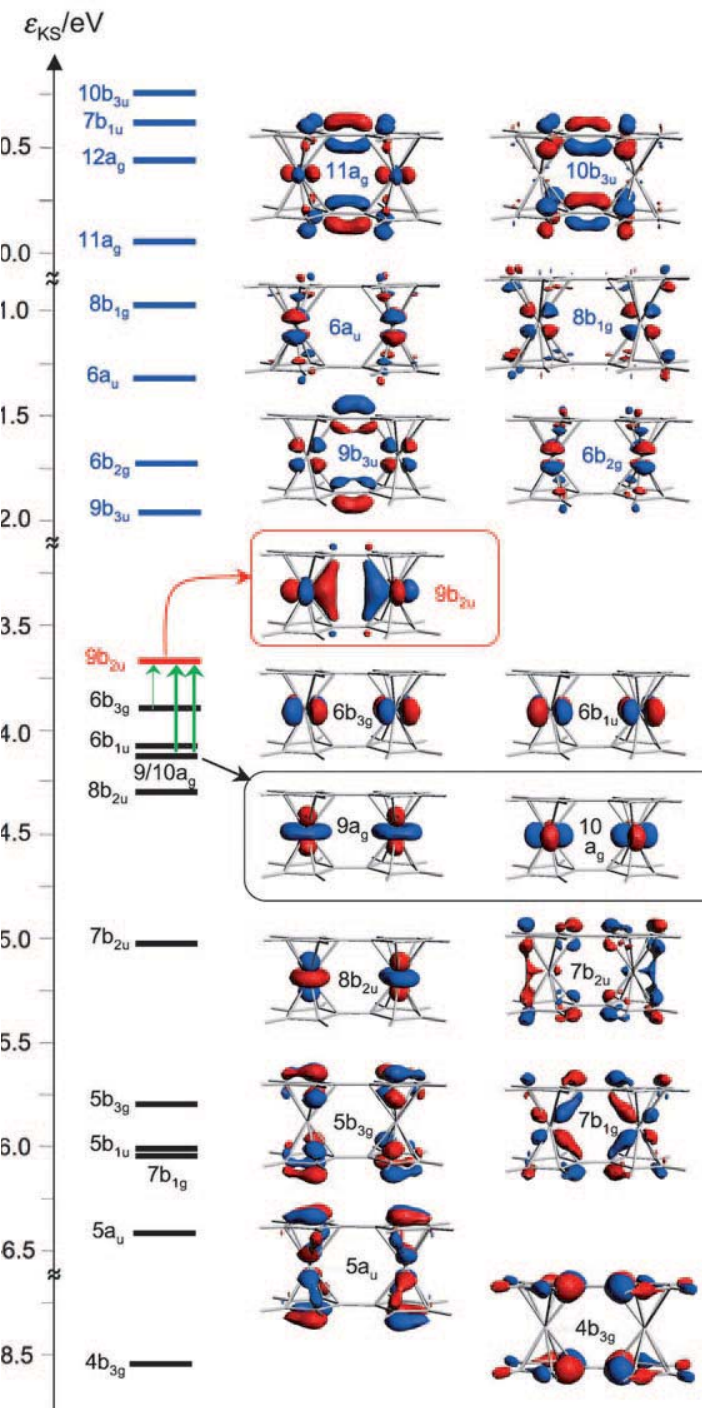

Figure 9. MO scheme of $\mathrm{Fc}=\mathrm{Fc}$ from BP86/DZ(ZORA) calculations with the ADF program. Blue levels are virtual MOs, red levels are open shells in the radical cations, and black levels are the doubly occupied MOs. Dipole-allowed IR transitions are indicated by green arrows. lower energy linear combinations of the Fc ligand $\pi$ MOs $4 \mathrm{e}_{1}^{\prime}$ and the Fc metal-ligand bonding MOs $3 \mathrm{e}_{1}^{\prime \prime}$ are found. At higher energy the unoccupied linear combinations of $\mathrm{Fc}$ $\mathrm{d}_{x z}$ and $\mathrm{d}_{y z}$ orbitals are located, which are slightly antibonding with respect to the Cp ligands. The LUMO $\left(9 b_{2 u}\right)$ derives from the out-of-phase combination of the $\mathrm{Fc} \mathrm{d}_{x z}$ orbitals. At even higher energy, ligand $\pi^{*}$ orbitals are located $\left(11 \mathrm{a}_{\mathrm{g}}, 10 \mathrm{~b}_{3 \mathrm{u}}\right)$.

Optical absorption spectroscopy: Figure 10 shows the absorption spectra of the doubly bridged ferrocenylene ferrocenylenium dimer (= bisfulvalenide diiron cation) $\mathrm{Fc}=\mathrm{Fc}^{\bullet+}$,

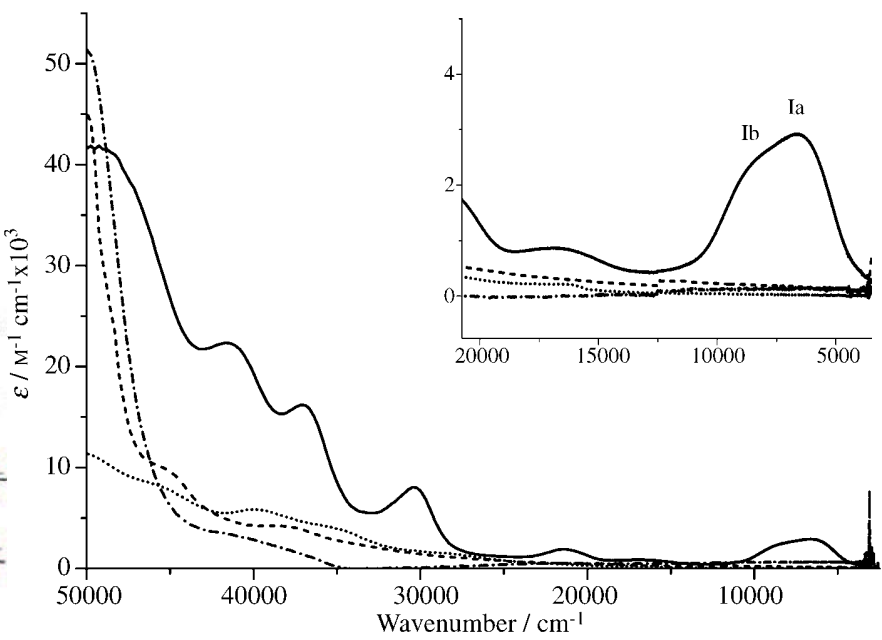

Figure 10. UV-visible-NIR-MIR spectra of $\mathrm{Fc}=\mathrm{FcPF}_{6}(-)$ along with those of the constituent subunits ferrocene $(\mathrm{Fc} ;-\cdot-\bullet)$ and ferrocenium hexafluorophosphate $\left(\mathrm{FcPF}_{6} ; \cdots \cdot\right)$ in acetonitrile and the neutral parent compound biferrocenylene $(\mathrm{Fc}=\mathrm{Fc} ;---)$ in $\mathrm{KBr}, 300 \mathrm{~K}$.

along with those of the parent neutral dimer $\mathrm{Fc}=\mathrm{Fc}$ and the constituent subunits ferrocene $(\mathrm{Fc})$ and ferrocenium hexafluorophosphate, $\mathrm{Fc}^{\cdot+} \mathrm{PF}_{6}^{-}$. As shown at a larger scale in the inset, an important new feature of the oxidized compound is the intense band peaking at $6500 \mathrm{~cm}^{-1}$ (band Ia), which exhibits a shoulder at approximately $8400 \mathrm{~cm}^{-1}$ (band Ib; cf. Table 6). These features correspond to the MIR/NIR bands of the singly bridged Fc dimers and have been interpreted

Table 6. Electronic absorption bands $\left(\tilde{v}\right.$ in $\left.\mathrm{cm}^{-1}\right)$ of $\mathrm{Fc}=\mathrm{Fc}^{\cdot+}$.

\begin{tabular}{|c|c|c|c|c|}
\hline \multirow{2}{*}{$\begin{array}{l}\text { Band } \\
\text { no. }\end{array}$} & \multicolumn{3}{|c|}{ Band position of sample in } & \multirow{2}{*}{$\begin{array}{l}\text { Assignment } \\
\text { of transition }^{[b]}\end{array}$} \\
\hline & $\mathrm{CH}_{3} \mathrm{CN}^{[\mathrm{a}]}$ & $\mathrm{KBr}$ pellet & solid $\mathrm{Ar} / 10 \mathrm{~K}$ & \\
\hline Ia & $6500(3000)$ & 6200 & 6500 & $\begin{array}{l}1^{2} A_{g}\left(10 a_{g} \beta \rightarrow 9 b_{2 u} \beta /\right. \\
\left.9 a_{g} \beta \rightarrow 9 b_{2 u} \beta\right)\end{array}$ \\
\hline \multirow[t]{5}{*}{$\mathrm{Ib}$} & $8400(2000)$ & 8200 & 8500 & $\begin{array}{l}2^{2} A_{g}\left(10 a_{g} \beta \rightarrow 9 b_{2 u} \beta /\right. \\
\left.9 a_{g} \beta \rightarrow 9 b_{2 u} \beta\right)\end{array}$ \\
\hline & $17000(1000)$ & - & $(17000)$ & $\begin{array}{l}{ }^{2} \mathrm{~B}_{3 \mathrm{~g}}\left(5 \mathrm{~b}_{3 \mathrm{~g}} \beta \rightarrow 9 \mathrm{~b}_{2 \mathrm{u}} \beta ;\right. \\
\text { ("hole transition") }\end{array}$ \\
\hline & $22000(2000)$ & - & 22000 & MLCT/LMCT \\
\hline & $30000(8000)$ & - & 30000 & $\begin{array}{l}4^{2} A_{g}\left(8 a_{g} \beta \rightarrow 9 b_{2 u} \beta\right. \\
\text { LMCT })\end{array}$ \\
\hline & $37000(16000)$ & & 37000 & CT \\
\hline
\end{tabular}

[a] $\varepsilon$ values are in brackets. [b] Refer to Figure 9 and Table 9 . 


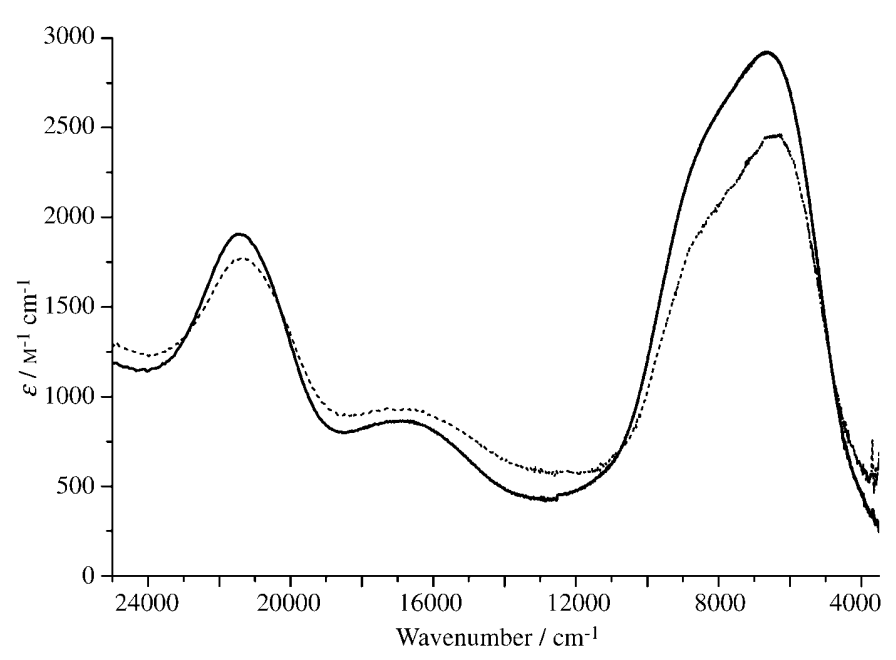

Figure 11. Visible-NIR spectra of $\mathrm{Fc}=\mathrm{FcPF}_{6}$ in acetonitrile $(-)$ and dichloromethane (----).

as IVCT transitions in the literature. ${ }^{[25]}$ This assignment is supported by the TD-DFT calculations, which attribute these bands to transitions from the doubly occupied metaltype orbitals to the SOMO $9 b_{2 u}$ (see below).

The solution spectra of $\mathrm{Fc}=\mathrm{Fc}^{\cdot+}$ in $\mathrm{CD}_{3} \mathrm{CN}$ and $\mathrm{CD}_{2} \mathrm{Cl}_{2}$ are compared in Figure 11. In contrast to the spectrum of the monobridged radical cations, bands Ia and Ib do not exhibit solvatochromic behavior. ${ }^{[45]}$ The spectrum in $\mathrm{KBr}$ shows these bands at 6200 and $8200 \mathrm{~cm}^{-1}$ (data not shown). As also evident from this figure, the temperature dependence of these bands in $\mathrm{KBr}$ pellets is less pronounced than in case of the $\mathrm{Fc}_{2}{ }^{+}$dimers; only a slight decrease in line width is observed upon lowering the temperature. At higher energy in the solution spectra (Figure 11) two medium-intense bands are found $\left(17000,22000 \mathrm{~cm}^{-1}\right)$, and at $30000 \mathrm{~cm}^{-1}$ the first high-intensity band appears. In any case, the transitions of the radical cation $\mathrm{Fc}=\mathrm{Fc}^{*+}$ are much more intense than those of its neutral counterpart.

To obtain more information about these transitions we also attempted matrix-isolation experiments with $\mathrm{Fc}=\mathrm{Fc}$. However, these experiments were less successful than in the above case of $\mathrm{Fc}_{2}$ in that an impurity of slightly higher volatility than $\mathrm{Fc}=\mathrm{Fc}$ was always present. Although this impurity showed no tangible absorption below $40000 \mathrm{~cm}^{-1}$, it gave rise to rather intense bands in the range of 20000$35000 \mathrm{~cm}^{-1}$ after X-ray irradiation (i.e., ionization). Therefore we had to resort to subtraction of the spectra obtained in two ionization experiments in which different amounts of $\mathrm{Fc}=\mathrm{Fc}$ were present in the matrix to obtain the spectrum of $\mathrm{Fc}=\mathrm{Fc}^{\cdot+}$ presented in Figure 12. This spectrum shows similar transitions as were found in the solution spectra of $\mathrm{Fc}=\mathrm{Fc}^{\cdot+}$, and in the following it will be used to assign the electronic transitions of $\mathrm{Fc}=\mathrm{Fc}^{\circ+}$, based on TD-DFT calculations.

TD-DFT calculations and band assignments: Tables 8 and 9 show the results of TD-DFT calculations on $\mathrm{Fc}=\mathrm{Fc}$ and $\mathrm{Fc}=$ $\mathrm{Fc}^{\cdot+}$, respectively. The calculated band positions and intensi-

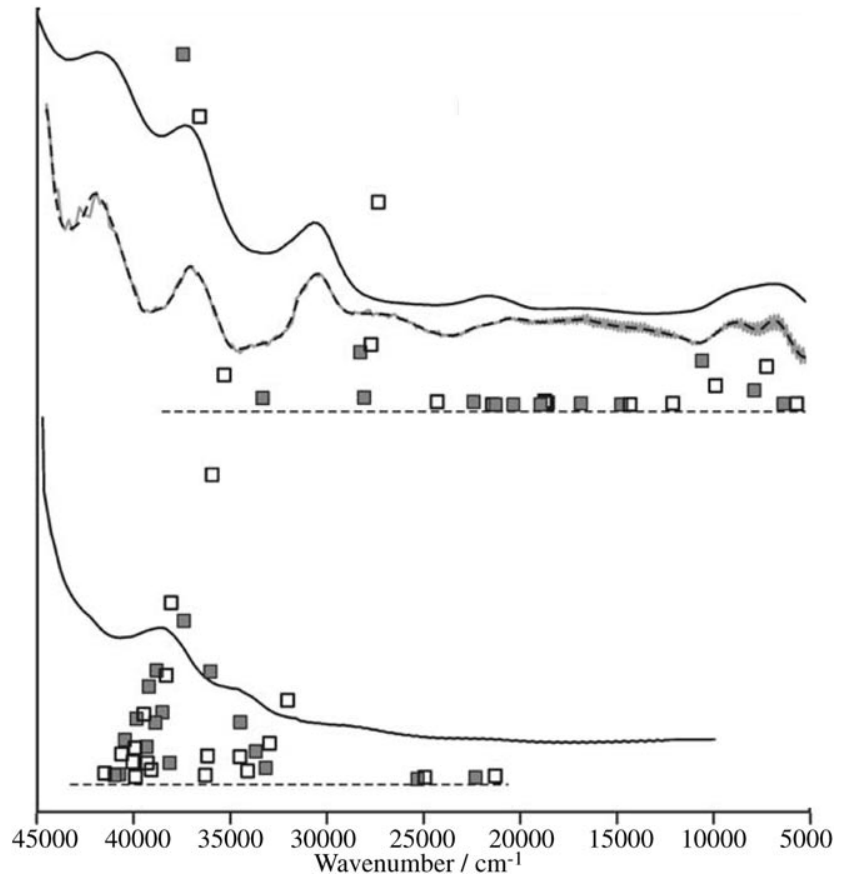

Figure 12. Electronic absorption spectra of $\mathrm{Fc}=\mathrm{Fc}$ in $\mathrm{Ar}$ at $10 \mathrm{~K}$ (bottom panel; - ) and $\mathrm{Fc}=\mathrm{Fc}_{2}{ }^{+}$in $\mathrm{Ar}$ at $10 \mathrm{~K}$ (top panel; ---) and in $\mathrm{CH}_{3} \mathrm{CN}$ (top panel; - ) The filled squares $(\square)$ denote electronic transitions predicted by TD-BP86 calculations using the TZP basis set and the ZORA method as implemented in the ADF program, whereas the open squares ( $\square$ ) refer to calculations using the LANL2DZ basis set with the Gaussian program, as listed in Tables 8 (for $\mathrm{Fc}=\mathrm{Fc}$ ) and 9 (for $\mathrm{Fc}=\mathrm{Fc}_{2}{ }^{+}$), respectively.

ties are also represented schematically in the matrix-isolation spectra of these compounds (Figure 12). The resulting assignments of experimentally observed bands are included in Tables 6 and 7. As is already evident from this figure, the situation is by far more complex for the bisfulvalenide diiron systems than for the singly bridged biferrocene dimers. Both for the neutral and the radical cation DFT predicts a multitude of densely spaced excited states out of which only a few, which are not dipole forbidden or are associated with vanishingly small transition dipole moments, are listed in the tables (120 states had to be calculated to reach $45000 \mathrm{~cm}^{-1}$ !).

Table 7. Electronic absorption bands $\left(\tilde{v}\right.$ in $\left.\mathrm{cm}^{-1}\right)$ of $\mathrm{Fc}=\mathrm{Fc}$.

\begin{tabular}{llll}
\hline $\begin{array}{l}\text { Band } \\
\text { no. }\end{array}$ & \multicolumn{2}{c}{ Band position of sample in } & $\begin{array}{l}\text { Assignment } \\
\text { solid } \mathrm{Ar} / 10 \mathrm{~K}\end{array}$ \\
\hline Ia & $21200(500)$ & 22000 (weak) & LF (mixed) \\
Ib & - & 28000 (shoulder) & LF/CT \\
II a & - & 35000 & $?$ \\
IIb & $39000(4000)$ & 38000 & $?$ \\
\hline
\end{tabular}

[a] $\varepsilon$ values are in brackets. [b] Refer to Figure 9 and Table 8.

In neutral $\mathrm{Fc}=\mathrm{Fc}$ it is difficult to reach definite assignments for the observed bands (dotted line in Figure 12). The 
Table 8. Electronic transitions $\left(\tilde{v}\right.$ in $\left.\mathrm{cm}^{-1}\right)$ of neutral $\mathrm{Fc}=\mathrm{Fc}$ calculated by the TD-BP86 method.

\begin{tabular}{|c|c|c|c|c|c|}
\hline \multirow[t]{2}{*}{ States } & \multicolumn{2}{|c|}{$\mathrm{ADF}^{[\mathrm{a}]}$} & \multicolumn{2}{|c|}{ Gaussian $03^{[\mathrm{b}]}$} & \multirow{2}{*}{$\begin{array}{l}\text { Configurational } \\
\text { composition }^{[\mathrm{c}]}\end{array}$} \\
\hline & $\tilde{v}\left[\mathrm{~cm}^{-1}\right]$ & $f$ & $\tilde{v}\left[\mathrm{~cm}^{-1}\right]$ & $f$ & \\
\hline $1^{2} \mathrm{~B}_{3 \mathrm{u}}$ & 22283 & 0.0018 & 21264 & 0.0013 & $\begin{array}{l}9 \mathrm{~b}_{2 \mathrm{u}} \rightarrow 8 \mathrm{~b}_{1 \mathrm{~g}}(0.68 / 0.60) \\
6 \mathrm{~b}_{3 \mathrm{~g}} \rightarrow 6 \mathrm{a}_{\mathrm{u}}(0.17 / 0.27) \\
6 \mathrm{~b}_{1 \mathrm{u}} \rightarrow 6 \mathrm{~b}_{2 \mathrm{~g}}(0.13 / 0.25)\end{array}$ \\
\hline $2^{2} \mathrm{~B}_{3 \mathrm{u}}$ & 25250 & 0.0004 & 24961 & 0.0011 & $\begin{array}{l}8 \mathrm{~b}_{2 \mathrm{u}} \rightarrow 8 \mathrm{~b}_{1 \mathrm{~g}}(0.28 / 0.34) \\
6 \mathrm{~b}_{1 \mathrm{u}} \rightarrow 6 \mathrm{~b}_{2 \mathrm{~g}}(0.25 / 0.30) \\
6 \mathrm{~b}_{3 \mathrm{~g}} \rightarrow 6 \mathrm{a}_{\mathrm{u}}(0.22 / 0.30) \\
9 \mathrm{~b}_{2 \mathrm{u}} \rightarrow 8 \mathrm{~b}_{1 \mathrm{~g}}(0.18 / 0.30)\end{array}$ \\
\hline $1^{2} \mathrm{~B}_{2 \mathrm{u}}$ & 32117 & 0.0187 & 32020 & 0.0188 & $\begin{array}{l}9 \mathrm{~b}_{2 \mathrm{u}} \rightarrow 11 \mathrm{a}_{\mathrm{g}}(0.68 / 0.58) \\
7 \mathrm{~b}_{1 \mathrm{~g}} \rightarrow 9 \mathrm{~b}_{3 \mathrm{u}}(-/ 0.37) \\
9 \mathrm{~b}_{2 \mathrm{u}} \rightarrow 12 \mathrm{a}_{\mathrm{g}}(0.21 /-)\end{array}$ \\
\hline $3^{2} \mathrm{~B}_{3 \mathrm{u}}$ & 33198 & 0.0031 & 32959 & 0.0089 & $\begin{array}{l}7 \mathrm{~b}_{2 \mathrm{u}} \rightarrow 8 \mathrm{~b}_{1 \mathrm{~g}}(0.61 / 0.61) \\
10 \mathrm{a}_{\mathrm{g}} \rightarrow 10 \mathrm{~b}_{3 \mathrm{u}}(0.37 / 0.30)\end{array}$ \\
\hline $4^{2} \mathrm{~B}_{3 \mathrm{u}}$ & 34586 & 0.0133 & 34508 & 0.0056 & $\begin{array}{l}10 \mathrm{a}_{\mathrm{g}} \rightarrow 10 \mathrm{~b}_{3 \mathrm{u}}(0.52 / 0.58) \\
7 \mathrm{~b}_{2 \mathrm{u}} \rightarrow 8 \mathrm{~b}_{1 \mathrm{~g}}(0.24 /-) \\
5 \mathrm{~b}_{3 \mathrm{~g}} \rightarrow 6 \mathrm{a}_{\mathrm{u}}(-/ 0.24)\end{array}$ \\
\hline $2^{2} \mathrm{~B}_{2 \mathrm{u}}$ & 33692 & 0.0070 & 35912 & 0.0707 & $\begin{array}{l}9 b_{2 u} \rightarrow 12 a_{g}(0.73 / 0.34) \\
7 b_{1 g} \rightarrow 9 b_{3 u}(-/ 0.35) \\
8 b_{2 u} \rightarrow 11 a_{g}(-/ 0.32)\end{array}$ \\
\hline $3^{2} \mathrm{~B}_{2 \mathrm{u}}$ & 35998 & 0.0245 & 36222 & 0.0057 & mixed \\
\hline $4^{2} \mathrm{~B}_{2 \mathrm{u}}$ & 37399 & 0.0356 & 37983 & 0.0410 & mixed \\
\hline $5^{2} \mathrm{~B}_{3 \mathrm{u}}$ & 38050 & 0.0042 & 38338 & 0.0247 & mixed \\
\hline $6^{2} \mathrm{~B}_{3 \mathrm{u}}$ & 38872 & 0.0132 & 39082 & 0.0029 & mixed \\
\hline $7^{2} \mathrm{~B}_{3 \mathrm{u}}$ & 40349 & 0.0094 & 39280 & 0.0047 & mixed \\
\hline $7^{2} \mathrm{~B}_{2 \mathrm{u}}$ & 39180 & 0.0211 & 39444 & 0.0153 & mixed \\
\hline
\end{tabular}

[a] BP86/TZP/ZORA. [b] BP86/LANL2DZ. [c] In terms of the MOs depicted in Figure 9. The coefficients of the CIS-type wave functions are from the (ADF/Gaussian) calculations, respectively.

Table 9. Electronic transitions $\left(\tilde{v}\right.$ in $\left.\mathrm{cm}^{-1}\right)$ of $\mathrm{Fc}=\mathrm{Fc}_{2}{ }^{+}$calculated by the TD-BP86 method.

\begin{tabular}{|c|c|c|c|c|c|}
\hline \multirow[t]{2}{*}{ States } & \multicolumn{2}{|c|}{$\mathrm{ADF}^{[\mathrm{a}]}$} & \multicolumn{2}{|c|}{ Gaussian $03^{[\mathrm{b}]}$} & \multirow{2}{*}{$\begin{array}{l}\text { Configurational } \\
\text { composition }^{[\mathrm{c}]}\end{array}$} \\
\hline & $\tilde{v}\left[\mathrm{~cm}^{-1}\right]$ & $f$ & $\tilde{v}\left[\mathrm{~cm}^{-1}\right]$ & $f$ & \\
\hline $1^{2} \mathrm{~B}_{3 \mathrm{~g}}$ & 6118 & $<10^{-4}$ & 5412 & 0.0001 & $6 b_{3 \mathrm{~g}} \beta \rightarrow 9 \mathrm{~b}_{2 \mathrm{u}} \beta(0.99 / 1.2)$ \\
\hline $1^{2} \mathrm{~A}_{\mathrm{g}}$ & 7635 & 0.0073 & 7125 & 0.0206 & $\begin{array}{l}10 \mathrm{a}_{\mathrm{g}} \beta \rightarrow 9 \mathrm{~b}_{2 \mathrm{u}} \beta(0.76 / 0.87) \\
9 \mathrm{a}_{\mathrm{g}} \beta \rightarrow 9 \mathrm{~b}_{2 \mathrm{u}} \beta(0.23 / 0.25)\end{array}$ \\
\hline $2^{2} \mathrm{~A}_{\mathrm{g}}$ & 10367 & 0.024 & 9732 & 0.0103 & $\begin{array}{l}9 \mathrm{a}_{\mathrm{g}} \beta \rightarrow 9 \mathrm{~b}_{2 \mathrm{u}} \beta(0.75 / 0.99) \\
10 \mathrm{a}_{\mathrm{g}} \beta \rightarrow 9 \mathrm{~b}_{2 \mathrm{u}} \beta(0.21 /-0.14)\end{array}$ \\
\hline $1^{2} \mathrm{~B}_{1 \mathrm{~g}}$ & 14520 & $<10^{-4}$ & 11900 & 0.0003 & $\begin{array}{l}10 \mathrm{a}_{\mathrm{g}} \beta \rightarrow 9 \mathrm{~b}_{3 \mathrm{u}} \beta(0.77 / 0.98) \\
10 \mathrm{a}_{\mathrm{g}} \alpha \rightarrow 9 \mathrm{~b}_{3 \mathrm{u}} \alpha(0.19 /-0.38)\end{array}$ \\
\hline $2^{2} \mathrm{~B}_{1 \mathrm{~g}}$ & 16616 & $<10^{-4}$ & 14209 & 0.0002 & $\begin{array}{l}9 \mathrm{a}_{\mathrm{g}} \beta \rightarrow 9 \mathrm{~b}_{3 \mathrm{u}} \beta(0.53 / 0.82) \\
10 \mathrm{a}_{\mathrm{g}} \alpha \rightarrow 9 \mathrm{~b}_{3 \mathrm{u}} \alpha(0.30 /-0.6)\end{array}$ \\
\hline $4^{2} B_{1 g}$ & 20180 & $<10^{-4}$ & 18466 & 0.0005 & $\begin{array}{l}10 \mathrm{a}_{\mathrm{g}} \alpha \rightarrow 9 \mathrm{~b}_{3 \mathrm{u}} \alpha(0.45 / 0.70) \\
9 \mathrm{a}_{\mathrm{g}} \beta \rightarrow 9 \mathrm{~b}_{3 \mathrm{u}} \beta(0.38 / 0.43)\end{array}$ \\
\hline $2^{2} \mathrm{~B}_{3 \mathrm{~g}}$ & 18750 & 0.0004 & 18473 & 0.0004 & $5 \mathrm{~b}_{3 \mathrm{~g}} \beta \rightarrow 9 \mathrm{~b}_{2 \mathrm{u}} \beta(0.99 / 1.00)$ \\
\hline $5^{2} B_{1 g}$ & 20944 & 0.0009 & 18473 & 0.0015 & $\begin{array}{l}9 \mathrm{a}_{\mathrm{g}} \alpha \rightarrow 9 \mathrm{~b}_{3 \mathrm{u}} \alpha(-/ 0.61) \\
9 \mathrm{a}_{\mathrm{g}} \beta \rightarrow 9 \mathrm{~b}_{3 \mathrm{u}} \beta(0.51 / 0.46) \\
7 \mathrm{~b}_{1 \mathrm{~g}} \beta \rightarrow 9 \mathrm{~b}_{2 \mathrm{u}} \beta(0.40 / 0.59)\end{array}$ \\
\hline $6^{2} \mathrm{~B}_{1 \mathrm{~g}}$ & 22213 & 0.0016 & 21135 & 0.0001 & $\begin{array}{l}7 b_{1 \mathrm{~g}} \beta \rightarrow 9 \mathrm{~b}_{2 \mathrm{u}} \beta(0.52 / 0.67) \\
9 \mathrm{a}_{\mathrm{g}} \alpha \rightarrow 9 \mathrm{~b}_{3 \mathrm{u}} \alpha(0.41 / 0.61)\end{array}$ \\
\hline $3^{2} \mathrm{~A}_{\mathrm{g}}$ & 27832 & 0.0039 & 24123 & 0.0017 & $\begin{array}{l}7 b_{1 \mathrm{~g}} \beta \rightarrow 9 \mathrm{~b}_{3 \mathrm{u}} \beta(0.49 / 0.77) \\
7 \mathrm{~b}_{1 \mathrm{~g}} \alpha \rightarrow 9 \mathrm{~b}_{3 \mathrm{u}} \alpha(0.43 / 0.73)\end{array}$ \\
\hline $4^{2} \mathrm{~A}_{\mathrm{g}}$ & 28073 & 0.0289 & 27487 & 0.0316 & $8 \mathrm{a}_{\mathrm{g}} \beta \rightarrow 9 \mathrm{~b}_{2 \mathrm{u}} \beta(0.95 / 0.97)$ \\
\hline $6^{2} \mathrm{~A}_{\mathrm{g}}$ & 33083 & 0.0039 & 35121 & 0.0163 & $\begin{array}{l}8 \mathrm{~b}_{2 \mathrm{u}} \beta \rightarrow 11 \mathrm{a}_{\mathrm{g}} \beta(-/ 0.79) \\
8 \mathrm{~b}_{2 \mathrm{u}} \alpha \rightarrow 11 \mathrm{a}_{\mathrm{g}} \alpha(-/ 0.45) \\
9 \mathrm{~b}_{2 \mathrm{u}} \beta \rightarrow 11 \mathrm{a}_{\mathrm{g}} \beta(0.67 /-)\end{array}$ \\
\hline $7 / 9^{2} \mathbf{A}_{g}$ & 37240 & 0.1878 & 36357 & 0.1542 & $\begin{array}{l}8 \mathrm{~b}_{2 \mathrm{u}} \alpha \rightarrow 11 \mathrm{a}_{\mathrm{g}} \alpha(0.03 / 0.49) \\
9 \mathrm{~b}_{2 \mathrm{u}} \alpha \rightarrow 11 \mathrm{a}_{\mathrm{g}} \alpha(0.21 / 0.46) \\
7 \mathrm{~b}_{1 \mathrm{~g}} \beta \rightarrow 9 \mathrm{~b}_{3 \mathrm{u}} \beta(0.29 / 0.36)\end{array}$ \\
\hline
\end{tabular}

[a] BP86/TZP/ZORA. [b] BP86/LANL2DZ. [c] In terms of the MOs depicted in Figure 9. The coefficients of the CIS-type wave functions are from the (ADF/Gaussian) calculations, respectively. two first excited states of ligand-field (LF) and mixed LF/ $\mathrm{CT}$ character are predicted to be in the visible region $\left(\approx 22000\right.$ and $\left.25000 \mathrm{~cm}^{-1}\right)$, and in fact two weak absorptions are observed at 22000 and $28000 \mathrm{~cm}^{-1}$ (Table 7). Above $30000 \mathrm{~cm}^{-1}$, the spectrum shows two bands, but the calculations do not allow us to assign those to individual transitions, as some twenty of them are predicted to occur in this region with nonvanishing transition moments.

For the radical cation $\mathrm{Fc}=\mathrm{Fc}^{\cdot+}$, on the other hand, the calculations predict three electric-dipole-allowed transitions in the near- to mid-IR range that involve excitation of an electron from one of the doubly occupied metal-centered MOs to the singly occupied $9 \mathrm{~b}_{2 \mathrm{u}}$ SOMO. The first of these transitions leads to a ${ }^{2} B_{3 g}$ excited state but its oscillator strength is too weak for this transition to be observed experimentally under our conditions. The following two transitions to two ${ }^{2} \mathrm{~A}_{\mathrm{g}}$ excited states that lie about $1 \mathrm{eV}$ above the ground state are more intense. The two methods qualitatively agree with respect to the intensities of these transitions (oscillator strengths $\approx 10^{-2}$ ) and their absolute and relative energies (energy difference $\approx 0.3 \mathrm{eV}$ ). Above $10000 \mathrm{~cm}^{-1}$ a number of ligand-field transitions $\left(\mathrm{d}_{x^{2}-y^{2}}, \mathrm{~d}_{z^{2}} \rightarrow \mathrm{d}_{x z}\right)$ of small intensity $\left(f_{\max } \approx 5 \times 10^{-4}\right)$ are predicted. Then, at approximately $18500 \mathrm{~cm}^{-1}$, the lowest-energy charge-transfer (CT) band is calculated $\left(f \approx 4 \times 10^{-4}\right)$, which is associated with a transition from the purely ligand-type orbital $5 b_{3 g}$ to the $9 b_{2 u}$ SOMO. The calculated intensity of this transition (which would correspond to the "hole transitions" of $\mathrm{Fc}^{+}$and $\mathrm{Fc}_{2}{ }^{+}$; vide supra) is, however, weak. The calculations then predict a number of further, weak CT transitions, the first transition of appreciable intensity being a CT transition from $8 \mathrm{a}_{\mathrm{g}}$ to the SOMO $9 b_{2 u}$ at $28000 \mathrm{~cm}^{-1}\left(4^{2} \mathrm{~A}_{\mathrm{g}}\right.$ state). We associate this transition with the intense CT band at $30000 \mathrm{~cm}^{-1}$, which is also present in the solution spectrum of $\mathrm{Fc}=\mathrm{Fc}^{\cdot+}$. Another high-intensity transition predicted at approximately $37000 \mathrm{~cm}^{-1}$ can also be associated with an intense band appearing at this energy in the solution and Ar matrix spectra of $\mathrm{Fc}=\mathrm{Fc}^{\cdot+}$. On the other hand, no definite statement can be made on the origin of the two bands observed for this cation in the visible region, namely, at 17000 and $22000 \mathrm{~cm}^{-1}$, as the calculations do not predict any absorption intensity in this range of the spectrum.

\section{Discussion}

In the preceding sections we presented the electronic structures and optical absorption spectra of the $\mathrm{Fe}^{\mathrm{II}}-\mathrm{Fe}^{\mathrm{III}}$ mixedvalent biferrocenyl $\left(\mathrm{Fc}_{2}{ }^{++} ; \mathbf{1}\right), 1^{\prime}, 1^{\prime \prime \prime}$-diethyl biferrocenyl $\left((\mathrm{FcEt}){ }_{2}{ }^{+} ; \mathbf{2}\right)$, and bisfulvalenide diiron $\left(\mathrm{Fe}=\mathrm{Fe}^{{ }^{+}} ; \mathbf{3}\right)$ cations. Band assignments were made on the basis of TD-DFT calculations performed for both radical cations and their neutral precursors. It was found that in the NIR and MIR range of the absorption spectra of the mixed-valent dimers $\mathbf{1}$ und $\mathbf{2}$ two new bands Ia and Ib appear that are absent in the spectra of ferrocene and ferrocenium as well as in neutral biferrocene and bisfulvalenide diiron. Although the correspond- 
ing NIR band Ib has been known for the $\mathrm{Fc}_{2}{ }^{+}$cation, the existence of the MIR band Ia has been overlooked so far, in spite of a number of investigations on the spectroscopic properties of these systems and their solvent and pressure dependence. ${ }^{[6,25,46-53]}$

The energetic position and exclusive occurrence in the mixed-valent systems $\mathrm{Fc}_{2}{ }^{++}$and $(\mathrm{FcEt})_{2}{ }^{++}$suggest an IVCT character of these low-energy transitions. In our calculations, however, the charge and spin are fully delocalized and the electronic transitions do not involve any charge transfer between the two units. This agrees with PE spectroscopic data on $\mathrm{Fc}_{2}{ }^{+}$, which indicate that this cation behaves as a class III mixed-valent system in the gas phase. ${ }^{[41]}$ For the lowenergy electronic transition within the symmetric/antisymmetric pair of MOs containing the SOMO of a radical, Badger and Brocklehurst had proposed the term "charge resonance" band some time ago. ${ }^{[54]}$ Although this term seems more appropriate for class III systems because it does not imply any transfer of charge, it has unfortunately never caught on with the community dealing with mixed-valence compounds, where all such transitions are referred to as intervalence charge transfer (IVCT) transitions, regardless of whether they occur in class I, II, or III systems. We will adhere to this convention with the understanding that for true class III systems there is no charge transfer and the notion of these bands as IVCT transitions is, strictly speaking, erroneous.

Investigation of the $\mathrm{Fc}_{2}{ }^{+}$spectrum in solution indicated that the two components of band I show different solvent shifts. Assignment of band I to one IVCT transition exhibiting a double-peak band shape can therefore be excluded. The two absorption features must rather be associated with different electronic transitions. Optical absorption spectra of $\mathrm{Fc}_{2}{ }^{++}$salts recorded in $\mathrm{KBr}$ pellets reveal the presence of an additional absorption band, which we ascribe to intermolecular charge-transfer transitions, analogously to mononuclear ferrocenium salts. In contrast, the Ar matrix-isolation spectrum is the only one that shows the inherent spectroscopic properties of $\mathrm{Fc}_{2}{ }^{+}$, because interaction with the medium can be neglected in a good first approximation and interactions with counterions are entirely absent in these experiments. Thus, our discussion of the electronic structure of isolated $\mathrm{Fc}_{2}{ }^{+}$is primarily based on this spectrum. Comparison with the bands in different solvents and in $\mathrm{KBr}$ pellets then allows the unambiguous identification of shifts and additional bands.

To obtain theoretical information on the nature of the electronic ground state and the lowest-energy excited states of $\mathrm{Fc}_{2}{ }^{+}$and $\mathrm{Fc}=\mathrm{Fc}^{\cdot+}$ cations, DFT calculations were performed. After some disappointing experiences with the B3LYP functional we turned to the BP86 functional, which we used with the LANL2DZ basis (as implemented in the Gaussian 03 program) and with a TZP basis set optimized for the ZORA approximation to the relativistic Dirac equation (as implemented in the ADF program). According to spin-unrestricted calculations performed with both programs, the SOMO of $\mathrm{Fc}_{2}{ }^{+}$is the in-phase combination of the $d_{x^{2}-y^{2}}$ orbitals. At slightly lower energy three other doubly occupied metal-centered MOs follow before the outof-phase combination of the $\mathrm{d}_{x^{2}-y^{2}}$ AOs and the out-of-phase combination of the $\mathrm{d}_{z^{2}}$ AOs appear. Electronic excitation can thus occur from the out-of-phase to the singly occupied in-phase combination of $\mathrm{d}_{x^{2}-y^{2}}$. According to the TD-DFT calculations this transition is associated with band Ia. Band $\mathrm{Ib}$, which lies about $2000 \mathrm{~cm}^{-1}$ higher in energy and is weaker than band Ia, could in principle originate from a symmetric/antisymmetric set of $\mathrm{d}_{x y}$ or $\mathrm{d}_{z^{2}}$ orbitals. However, only one transition from the $\mathrm{d}_{z^{2}}$ combinations to the SOMO is electric-dipole allowed with a polarization similar to the $\mathrm{d}_{x^{2}-y^{2}} \rightarrow \mathrm{d}_{x^{2}-y^{2}}$ transition. We therefore assigned the second band to a $d_{z^{2}} \rightarrow d_{x^{2}-y^{2}}$ excitation. The transition is electricdipole allowed, but orbitally forbidden, and gets intensity by orbital mixing between $\mathrm{d}_{x^{2}-y^{2}}$ and $\mathrm{d}_{z^{2}}$. The $\mathrm{d}_{x y} \rightarrow \mathrm{d}_{x^{2}-y^{2}}$ transition, in contrast, is polarized perpendicular to the metalmetal axis and cannot acquire IVCT intensity in this way.

Comparison between the TD-DFT results and the measured spectra also allowed the assignment of some of the bands at higher energy for the biferrocene systems. For neutral $\mathrm{Fc}_{2}$ the HOMO-LUMO transition is calculated to be at $20000 \mathrm{~cm}^{-1}$ (obsd: $23000 \mathrm{~cm}^{-1}$ ); further LF states are predicted up to $28500 \mathrm{~cm}^{-1}$, but cannot be identified in the spectrum. Nevertheless, this calculated range corresponds to mononuclear ferrocene for which LF bands are observed in the range between 21800 and $30000 \mathrm{~cm}^{-1} \cdot{ }^{[42]}$ For neutral $\mathrm{Fc}_{2}$ transitions two charge-transfer states are calculated at 31000 (medium intense) and $33000 \mathrm{~cm}^{-1}$ (strong), in good agreement with the observed spectrum. These transitions are mainly of LMCT type (in ADF, the lower-energy transition also has some MLCT character), but are definitely lower in energy than in mononuclear ferrocene where the lowerenergy tail of the CT region is at approximately $37000 \mathrm{~cm}^{-1}$ ("band IV"). [42]

For cationic $\mathrm{Fc}_{2}{ }^{+}$, on the other hand, some of the bands in the UV-visible region could be assigned as well. The double-humped band II at $15000 / 18000 \mathrm{~cm}^{-1}$ most probably is associated with the SOMO-LUMO transition and the charge-transfer transition from the highest occupied ligand orbital to the metal-type SOMO. The latter would correspond to the so-called hole transition, which is observed at approximately $16000 \mathrm{~cm}^{-1}$ in $\mathrm{Fc}^{+}$salts. The SOMO-LUMO transition, calculated to occur at approximately $21000 \mathrm{~cm}^{-1}$, is the only ligand-field (LF) transition of appreciable intensity; other LF transitions (which in $\mathrm{Fc}^{+}$range from 17700 to $26300 \mathrm{~cm}^{-1}$ ) cannot be identified in the spectrum. At higher energy $\left(\approx 27000 \mathrm{~cm}^{-1}\right)$ a medium-intense absorption (band III) is found in the spectrum of $\mathrm{Fc}_{2}{ }^{+}$. This feature is probably associated with LMCT transitions, which again appear at lower energy than in the constituents $\mathrm{Fc}^{+}\left(>35300 \mathrm{~cm}^{-1}=\right.$ "band VI") and Fc (>37700= "band IV"). ${ }^{[42]}$

For comparison, the spectra of the bisfulvalenide diiron cation $\mathrm{Fc}=\mathrm{Fc}^{\cdot+}(\mathbf{3})$ were also recorded and analyzed with the help of DFT and TD-DFT calculations. Importantly, $\mathbf{3}$ also exhibits a low-energy double-peak IVCT transition, but, in contrast to the singly bridged $\mathrm{Fc}$ dimers, this feature 
has been known in the literature and interpreted as two transitions, the second one also being assigned as leading to a $\mathrm{d}_{z^{2}}$ exited state. ${ }^{[6]}$ Due to the larger bonding-antibonding interaction between the $\mathrm{d}_{x^{2}-y^{2}}$ MOs in the doubly bridged dimer, both IVCT transitions are shifted to higher energy and thus are easier to detect spectroscopically. TD-DFT allows the assignment of these two bands to excitations from the $d_{x^{2}-y^{2}}$ and $d_{z^{2}}$ type in-phase combinations $9 a_{\mathrm{g}}$ and $10 \mathrm{a}_{\mathrm{g}}$ to the singly occupied out-of-phase $\mathrm{d}_{x^{2}-y^{2}}$ combination $9 b_{2 u}$, which forms the SOMO of the complex. Identification of other LF transitions is difficult, both in the spectrum of neutral bisfulvalenide and the radical cation. The only transition of appreciable intensity in the visible region of the $\mathrm{Fc}=\mathrm{Fc}^{\cdot+}$ spectrum is predicted at $28000 \mathrm{~cm}^{-1}$, which is probably associated with the intense CT band at $30000 \mathrm{~cm}^{-1}$ in the solution spectrum of this cation. Another high-intensity transition predicted at $37000 \mathrm{~cm}^{-1}$ may also be associated with an intense band appearing at this energy in the solution and Ar matrix spectra of $\mathrm{Fc}=\mathrm{Fc}^{\bullet+}$.

In contrast to the IVCT transitions of the singly bridged dimers $\mathbf{1}$ and $\mathbf{2}$, the analogous transitions in the bisfulvalenide dimer 3 exhibit no solvent shifts. The solvent shift observed for band $\mathrm{Ib}$ in the spectra of the singly bridged ferrocene dimer radical cations, $\mathbf{1}$ and $\mathbf{2}$, indicates either 1) that in polar solvents spin and charge localize to some degree in one of the two Fc moieties, that is, that the charge-resonance transition acquires some charge-transfer character, and thus, becomes sensitive to solvent polarity, or 2) that the excited state that is attained in this transition is subject to a "sudden-polarization" effect, such as that occurring upon the twisting of totally symmetric excited states of polyenes. ${ }^{[55]}$ Information from other spectroscopic studies suggest that the first explanation applies in this case, that is, that bisferrocenyl cations are class II/III borderline cases ${ }^{[56]}$ in solution and in the solid state. More information on this issue is provided by the results of a detailed band shape analysis of the IVCT transitions Ia and Ib of compounds $\mathbf{1}$, $\mathbf{2}$, and $\mathbf{3}$, which is presented in another paper. ${ }^{[28]}$

\section{Conclusion}

We have presented the electronic structures and optical absorption spectra of the $\mathrm{Fe}^{\mathrm{II}}-\mathrm{Fe}^{\mathrm{III}}$ mixed-valent biferrocenyl (1), 1', 1'"'-diethyl biferrocenyl (2), and bisfulvalenide diiron (3) cations. Band assignments were made on the basis of TD-DFT calculations performed for both radical cations and their neutral precursors. It was found that in the NIR and MIR range of the absorption spectra of $\mathbf{1}$ und $\mathbf{2}$, two bands Ia and Ib appear that have not been reported before, and that are absent in the spectra of ferrocene, ferrocenium, neutral biferrocene, and bisfulvalenide diiron.

The new absorption feature and the known NIR band have both been assigned to IVCT transitions. Based on the molecular orbital scheme of cation $\mathbf{1}$ obtained with the BP86 functional and TD-DFT calculations, one band corresponds to the transition between the highest occupied $\mathrm{d}_{x^{2}-y^{2}}$ orbitals on the two iron centers, whereas the other one has been assigned to a transition from a lower-lying $d_{z^{2}}$ orbital to the $\mathrm{d}_{x^{2}-y^{2}}$ orbital.

In contrast to the IVCT transitions of dimers $\mathbf{1}$ and $\mathbf{2}$, the analogous transitions in dimer $\mathbf{3}$ exhibit no solvent shifts. It was deduced that there is a strong delocalization in compound $\mathbf{3}$, and that the monobridged compounds $\mathbf{1}$ and $\mathbf{2}$ either localize to some degree in one of the two Fc moieties or that the excited state is subject to a sudden-polarization effect.

\section{Acknowledgements}

F.T. acknowledges support of this research by the Christian Albrechts University of Kiel. The authors thank Prof. Herwig Schottenberger, Universität Innsbruck, for a sample of bisfulvalenide diiron. The work in Fribourg was supported by a grant of the Swiss National Science Foundation (project no. 200020-113268), which is gratefully acknowledged by T.B. and H.A.

[1] S. Barlow, D. O'Hare, Chem. Rev. 1997, 97, 637.

[2] D. N. Hendrickson, NATO ASI Ser. Ser. C 1991, 343, 67.

[3] H. Sano, Hyperfine Interact. 1990, 53, 97.

[4] T.-Y. Dong, L.-S. Chang, G.-H. Lee, S.-M. Peng, Organometallics 2002, 21, 4192.

[5] T. Oda, S. Nakashima, T. Okuda, Inorg. Chem. 2003, 42, 5376.

[6] W. H. Morrison, Jr., D. N. Hendrickson, Inorg. Chem. 1975, 14, 2331.

[7] S. C. Jones, S. Barlow, D. O'Hare, Chem. Eur. J. 2005, 11, 4473.

[8] D. E. Richardson, H. Taube, Coord. Chem. Rev. 1984, 60, 107.

[9] S. Iijima, R. Saida, I. Motoyama, H. Sano, Bull. Chem. Soc. Jpn. 1981, 54, 1375.

[10] S. Nakashima, A. Nishimori, Y. Masuda, H. Sano, M. Sorai, J. Phys. Chem. Solids 1991, 52, 1169.

[11] K. Boukheddaden, J. Linares, F. Varret, Phys. Rev. B 1993, 47, 14070.

[12] T.-Y. Dong, D. N. Hendrickson, K. Iwai, M. J. Cohn, S. J. Geib, A. L. Rheingold, H. Sano, I. Motoyama, S. Nakashima, J. Am. Chem. Soc. 1985, 107, 7996 .

[13] T. Kambara, D. N. Hendrickson, T.-Y. Dong, M. J. Cohn, J. Chem. Phys. 1987, 86, 2362.

[14] K. Boukheddaden, J. Linares, F. Varret, Phys. Rev. B 1994, 49, 15659.

[15] S. Klokishner, J. Linares, F. Varret, Chem. Phys. 1998, 226, 171.

[16] M. B. Robin, P. Day, Adv. Inorg. Chem. Radiochem. 1967, 9, 247.

[17] K. Y. Wong, P. N. Schatz, Prog. Inorg. Chem. 1981, 28, 369.

[18] T.-Y. Dong, T. Kambara, D. N. Hendrickson, J. Am. Chem. Soc. 1986, 108, 5857.

[19] T.-Y. Dong, T. Kambara, D. N. Hendrickson, J. Am. Chem. Soc. 1986, 108, 4423 .

[20] M. Sorai, A. Nishimori, D. N. Hendrickson, T. Y. Dong, M. J. Cohn, J. Am. Chem. Soc. 1987, 109, 4266.

[21] M. Konno, S. Kyodo, S. Iijima, Bull. Chem. Soc. Jpn. 1982, 55, 2327.

[22] B. S. Brunschwig, C. Creutz, N. Sutin, Chem. Soc. Rev. 2002, 31, 168.

[23] P. N. Schatz in Inorganic Electronic Structure and Spectroscopy (Eds.: E. I. Solomon, A. B. P. Lever), Wiley, New York, 1999.

[24] S. B. Piepho, E. R. Krausz, P. N. Schatz, J. Am. Chem. Soc. 1987, 109, 2996.

[25] D. R. Talham, D. O. Cowan, Organometallics 1984, 3, 1712.

[26] C. LeVanda, K. Bechgaard, D. O. Cowan, U. T. Mueller-Westerhoff, P. Eilbracht, G. A. Candela, R. L. Collins, J. Am. Chem. Soc. 1976, 98, 3181.

[27] U. T. Mueller-Westerhoff, P. Eilbracht, J. Am. Chem. Soc. 1972, 94, 9272. 
[28] R. Warratz, F. Tuczek, unpublished results.

[29] K. Yamakawa, M. Hisatome, Y Sako, S. Ichida, J. Organomet. Chem. 1975, 93, 219.

[30] K. E. Schwarzhans, H. Schottenberger, Z. Naturforsch. 1983, 38b, 1493.

[31] T. Bally in Reactive Intermediate Chemistry (Eds.: R. A. Moss, M. S. Platz, M. Jones), Wiley, New York 2005.

[32] A. D. Becke, Phys. Rev. A 1988, 38, 3098.

[33] J. P. Perdew, Phys. Rev. B 1986, 33, 8822.

[34] Z.-F. Xu, Y. Xie, W.-L. Feng, H. F. J. Schaefer, J. Phys. Chem. A 2003, 107, 2716.

[35] Gaussian 03 Revision C01, M. J. Frisch, G. W. Trucks, H. B. Schlegel, G. E. Scuseria, M. A. Robb, J. R. Cheeseman, J. A. Montgomery, Jr., T. Vreven, K. N. Kudin, J. C. Burant, J. M. Millam, S. S. Iyengar, J. Tomasi, V. Barone, B. Mennucci, M. Cossi, G. Scalmani, N. Rega, G. A. Petersson, H. Nakatsuji, M. Hada, M. Ehara, K. Toyota, R. Fukuda, J. Hasegawa, M. Ishida, T. Nakajima, Y. Honda, O. Kitao, H. Nakai, M. Klene, X. Li, J. E. Knox, H. P. Hratchian, J. B. Cross, C. Adamo, J. Jaramillo, R. Gomperts, R. E. Stratmann, O. Yazyev, A. J. Austin, R. Cammi, C. Pomelli, J. W. Ochterski, P. Y. Ayala, K. Morokuma, G. A. Voth, P. Salvador, J. J. Dannenberg, V. G. Zakrzewski, S. Dapprich, A. D. Daniels, M. C. Strain, O. Farkas, D. K. Malick, A. D. Rabuck, K. Raghavachari, J. B. Foresman, J. V. Ortiz, Q. Cui, A. G. Baboul, S. Clifford, J. Cioslowski, B. B. Stefanov, G. Liu, A. Liashenko, P. Piskorz, I. Komaromi, R. L. Martin, D. J. Fox, T. Keith, M. A. Al-Laham, C. Y. Peng, A. Nanayakkara, M. Challacombe, P. M. W. Gill, B. Johnson, W. Chen, M. W. Wong, C. Gonzalez, J. A. Pople, Gaussian, Inc., Wallingford CT, 2004.

[36] T. H. Dunning, Jr., P. J. Hay in Modern Theoretical Chemistry (Ed.: H. F. Schaefer III), Plenum, New York, 1976.

[37] P. J. Hay, W. R. Wadt, J. Chem. Phys. 1985, 82, 270, 284-299.

[38] ADF2004.01, SCM, E. J. Baerends. J. Autschbach, A. Bérces, C. Bo, P. M. Boerrigter, L. Cavallo, D. P. Chong, L. Deng, R. M. Dickson, D. E. Ellis, L. Fan, T. H. Fischer, C. Fonseca Guerra, S. J. A. van Gisbergen, J. A. Groeneveld, O. V. Gritsenko, M. Gruening, F. E. Harris, P. van den Hoek, H. Jacobsen, G. van Kessel, F. Kootstra, E. van Lenthe, D. A. McCormack, V. P. Osinga, S. Patchkovskii, P. H. T. Philipsen, D. Post, C. C. Pye, W. Ravenek, P. Ros, P. R. T. Schipper, G. Schreckenbach, J. G. Snijders, M. Sola, M. Swart, D.
Swerhone, G. te Velde, P. Vernooijs,L. Versluis, O. Visser, E. van Wezenbeek, G. Wiesenekker, S. K. Wolff, T. K. Woo, T. Ziegler, Theoretical Chemistry, Vrije Universiteit, Amsterdam, TheNetherlands, http://www.scm.com

[39] a) E. van Lenthe, E. J. Baerends, J. Snijders, J. Chem. Phys. 1993, 99 4597; b) E. van Lenthe, E. J. Baerends, J. Snijders, J. Chem. Phys. 1996, 105,6505 .

[40] M. E. Casida in Recent Advances in Density Functional Methods, part I (Ed.: D. P. Chong), World Scientific, Singapore, 1995, p. 155.

[41] D. L. Lichtenberger, H.-J. Fan, N. E. Gruhn, J. Organomet. Chem. 2003, 666, 75 .

[42] H. B. Gray, Y. S. Sohn, D. N. Hendrickson, J. Am. Chem. Soc. 1971 93, 3603.

[43] P. Brüggeller, P. Jaitner, H. Schottenberger, K. E. Schwarzhans, J. Organomet. Chem. 1991, 417, C53.

[44] M. Hillman, A. Kvick, Organometallics 1983, 2, 1780.

[45] G. E. McManis, R. M. Nielson, M. J. Weaver, Eur. J. Inorg. Chem. 1988, 27, 1827

[46] D. O. Cowan, F. Kaufman, J. Am. Chem. Soc. 1970, 92, 219.

[47] D. O. Cowan, F. Kaufman, J. Am. Chem. Soc. 1970, 92, 6198

[48] D. O. Cowan, C. LeVanda, J. Am. Chem. Soc. 1972, 94, 9271

[49] C. LeVanda, K. Bechgaard, D. O. Cowan, J. Org. Chem. 1976, 41, 2700.

[50] M. J. Powers, T. J. Meyer, J. Am. Chem. Soc. 1978, 100, 4393.

[51] M. D. Lowery, W. S. Hammack, H. G. Drickamer, D. N. Hendrickson, J. Am. Chem. Soc. 1987, 109, 8019.

[52] U. Sinha, M. D. Lowery, W. S. Hammack, D. N. Hendrickson, H. G. Drickamer, J. Am. Chem. Soc. 1987, 109, 7340.

[53] U. Sinha, M. D. Lowery, W. L. Walter, H. G. Drickamer, D. N. Hendrickson, J. Am. Chem. Soc. 1988, 110, 2471.

[54] B. Badger, B. Brocklehurst, Trans. Faraday Soc. 1969, 65, 2576.

[55] a) A. Viel, R. P. Krawczyk, U. Manthe, W. Domcke, Angew. Chem. 2003, 115, 3556; Angew. Chem. Int. Ed. 2003, 42, 3434, and references therein; b) V. Bonačić-Koutecký, J. Čižek, D. Döhnert, J. Koutecký, J. Chem. Phys. 1978, 69, 1168

[56] D. M. Alessandro, F. R. Keene, Chem. Soc. Rev. 2006, 35, 393. 